\title{
Fundamentals of Multiferroic Materials and Their Possible Applications
}

\author{
Melvin M. Vopson ${ }^{1}$ \\ University of Portsmouth, Faculty of Science, SEES, Burnaby Building, Portsmouth PO1 3QL, United Kingdom
}

\begin{abstract}
Materials science is recognized as one of the main factors driving development and economic growth. Since the silicon industrial revolution of the 1950s, research and developments in materials and solid state science have radically impacted and transformed our society by enabling the emergence of the computer technologies, wireless communications, Internet, digital data storage and widespread consumer electronics. Today's emergent topics in solid state physics such as nano-materials, graphene and carbon nano-tubes, smart and advanced functional materials, spintronic materials, bio-materials and multiferroic materials, promise to deliver a new wave of technological advances and economic impact, comparable to the silicon industrial revolution of the 1950s.

The surge of interest in multiferroic materials over the past 15 years has been driven by their fascinating physical properties and huge potential for technological applications. This review article addresses some of the fundamental aspects of solid-state multiferroic materials, followed by the detailed presentation of the latest and most interesting proposed applications of these multifunctional solid-state compounds. The applications presented here are critically discussed in the context of the state-of-the-art and current scientific challenges. They are highly interdisciplinary covering a wide range of topics and technologies including sensors, microwave devices, energy harvesting, photovoltaic technologies, solid-state refrigeration, data storage recording technologies and random access multi-state memories. According to their potential and expected impact, it is estimated that multiferroic technologies could soon reach multibillion US dollar market value.
\end{abstract}

Keywords: multiferroic materials, applications of multiferroics, physics of multiferroic materials

\section{Multiferroics: brief introduction and definitions}

Multiferroic materials are a special class of solid-state compounds, in which at least two order states such as magnetic, electric or piezo-elastic phases coexist. Therefore, well-known materials such as ferromagnets displaying strong piezo-magnetic properties, as well as piezoferroelectrics are arguably a special class of magneto-elastic or electro-elastic coupled multiferroics. In both cases, these materials are extremely important for technological applications because the coupling between the ferro-phases and the piezo-elastic properties facilitates a direct control of ferromagnetic and ferroelectric properties via externally applied mechanical stress. In addition, converse effects, in which externally applied magnetic and electric fields can modify the shape of the material by inducing a mechanical strain, exist.

This complex coupling between electric polarization - electric field - strain, magnetization magnetic field - strain and stress - strain - electric polarization - magnetization is graphically illustrated in figure 1.

While the coexistence of ferro-order and piezo-elastic properties can give rise to interesting piezo-electric and piezo-magnetic couplings, there are two additional multiferroic possible states, each displaying cross couplings between the order states. These additional types of multiferroics are the subject of this review article and they are: i) magneto-electric multiferroic, in which the electric and magnetic order states coexist; ii) elasto-magnetoelectric multiferroic, in which all three order states are present. It is important to mention that,

${ }^{1}$ Formerly known as M. Vopsaroiu. Correspondence: melvin.vopson@ port.ac.uk 
by electric and magnetic order state it is understood any type of ferroic ordering including ferromagnetic, anti-ferromagnetic, ferri-magnetic, paramagnetic, super-paramagnetic and their electrical equivalents, if applicable.

However, what makes multiferroic materials scientifically and technologically fascinating is not only their ability to display multiple order states but, most importantly, the cross coupling effects that can occur between the order states.

Besides the piezo-ferro couplings, an equally interesting and useful property of some multiferroics is the so-called "magneto-electric" coupling, which occurs in materials where magnetic - electric or magnetic-electric-elastic phases coexist. The magneto-electric effect facilitates the conversion between energies stored in magnetic and electric fields. Hence, magneto-electric multiferroic materials have the unique property that when subjected to an applied external magnetic field, the electric polarization is modified and, conversely, the

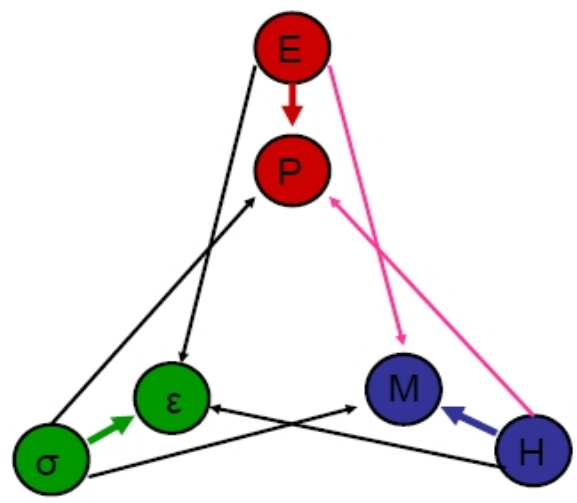

Figure 1. Possible cross-couplings in multiferroics. E - electric field; $\mathbf{P}$ electric polarization; $\sigma$ - applied mechanical stress; $\varepsilon$ - strain; H magnetic field; $\mathbf{M}$ - magnetization application of an external electric field alters the net magnetization of the multiferroic solid. The magneto-electric effect in multiferroics can therefore be electrically or magnetically induced and it is mathematically described by the magneto-electric coupling coefficient, $\alpha$ [15]. The electrically induced magneto-electric effect is defined as the change in the magnetization $(\mathrm{M})$ of the sample due to the application of an electric field (E):

$$
\alpha_{E}=\left(\frac{\partial M}{\partial E}\right)
$$

Similarly, the magnetically induced magneto-electric effect is the change in the electric polarization $(\mathrm{P})$ of the sample due to the application of a magnetic field $(\mathrm{H})$ :

$$
\alpha_{H}=\left(\frac{\partial P}{\partial H}\right) \cong \varepsilon_{0} \varepsilon_{r}\left(\frac{\partial E}{\partial H}\right)
$$

where $\varepsilon_{0}$ and $\varepsilon_{\mathrm{r}}$ are the dielectric permitivity of the vacuum and the relative permitivity of the medium, respectively and in (2) it has been assumed that $\varepsilon_{\mathrm{r}} \gg 1\left(\mathrm{P}=\varepsilon_{0} \chi \mathrm{E}=\varepsilon_{0}\left(\varepsilon_{\mathrm{r}}-1\right) \mathrm{E} \approx\right.$ $\varepsilon_{0} \varepsilon_{\mathrm{r}} \mathrm{E}$ for $\left.\varepsilon_{\mathrm{r}} \gg 1\right)$. Since $\mathrm{E}=\mathrm{V} / \mathrm{t}$, where $\mathrm{V}$ is the voltage and $\mathrm{t}$ is the thickness of the dielectric structure, the following relation for the magnetically induced magneto-electric effect is obtained:

$$
\alpha_{H}=\left(\frac{\partial P}{\partial H}\right) \cong \varepsilon_{0} \varepsilon_{r}\left(\frac{\partial E}{\partial H}\right)=\frac{\varepsilon_{0} \varepsilon_{r}}{t}\left(\frac{\partial V}{\partial H}\right)=\alpha_{H}{ }^{V} \varepsilon_{0} \varepsilon_{r}
$$

where $\alpha_{H}{ }^{v}$ is the magnetically induced voltage magneto-electric coefficient defined as:

$$
\alpha_{H}{ }^{V}=\left(\frac{\partial E}{\partial H}\right)=\frac{1}{t}\left(\frac{\partial V}{\partial H}\right)
$$


The voltage magneto-electric coefficient is the main parameter used in analyzing experimental measurements and designing various applications based on multiferroics. The relationship between the magnetically induced magneto-electric coupling coefficient and the voltage magneto-electric coefficient is: $\alpha_{H}=\varepsilon_{0} \cdot \varepsilon_{\mathrm{r}} \cdot \alpha_{\mathrm{H}}{ }^{\mathrm{V}}$ [6]. Both $\alpha_{\mathrm{H}}$ and $\alpha_{\mathrm{E}}$ are expressed in $[\mathrm{s} / \mathrm{m}]$ in SI units. However, the more practical voltage magneto-electric coefficient, $\alpha_{\mathrm{H}}{ }^{\mathrm{V}}$ (see relation (4)) is expressed as [V/A] in SI units and as [V/cm.Oe] in CGS units, which are also utilized in most practical applications and scientific measurements [7,8].

There are two possible types of magneto-electric coupling: direct and indirect. The direct magneto-electric coupling refers to multiferroics in which the direct application of electric or magnetic field results in changes of magnetization or electric polarization of the system, respectively. This effect is characteristic to a special class of multiferroic materials called "single-phase" multiferroics [9].

A second mechanism of activating the magneto-electric effect occurs primarily in elastomagneto-electric multiferroics, also known as composite multiferroics $[8,10]$, and it is called the indirect magneto-electric effect. In the case of indirect magneto-electric effect, the application of magnetic or electric field does not affect directly the electric polarization or magnetization, respectively. Instead, they are modified indirectly via a strain mediated magneto-electric coupling [11].

\section{Multiferroics: historical and present developments}

It is believed that Schmid first introduced the term "multiferroic" in 1994 [12]. Although the term multiferroic is rather recent, multiferroic materials and the possibility of magnetoelectric coupling in solids was first predicted by Curie in 1894 based on crystal symmetry considerations [13], while the term magneto-electric coupling was first coined by Debye in 1926 [14]. Pioneering work on magneto-electric effect in multiferroic materials goes back to more than half a century. In 1959, Dzyaloshinskii was the first to report a magneto-electric effect in anti-ferromagnetic $\mathrm{Cr}_{2} \mathrm{O}_{3}$ dielectrics [15], followed by similar experimental and theoretical studies published by Astrov, Folen, Stader and Rado [1-3]. O'Dell summarized most of the theoretical and experimental achievements in the field of magneto-electric media up to 1970 in an article published in 1962 [16] and a book published in 1970 [17]. Following these exiting developments, the interest in magneto-electric multiferroics grew substantially because of the realization of their potential for technological applications. This culminated with the publication by Wood and Austin in 1973 of the only article reviewing possible applications of multiferroics at that time [18], followed by a recent short review published by Scott in 2012 and briefly covering applications published in the 2009 - 2012 timeframe [19]. However, after the initial surge in interest in the field of magneto-electric multiferroics, the topic has lost momentum with the scientific community. The main reasons were the fact that despite intensive research in this field, very few magneto-electric multiferroic compounds were discovered. The already known or synthesized multiferroics displayed very small magneto-electric coupling effect and usually this was manifested only at cryogenic temperatures. These features made mutiferroics nothing more than an interesting subject for academic research, since most useful applications require room temperature operation and large magneto-electric coupling. Moreover, this was complemented by a limited understanding of the microscopic nature of multiferroicity and magneto-electric coupling, which led unsurprisingly to a decline in multiferroics research activities for almost 20 years. 
For example, according to the Web of Science (WoS), since 1970 until 2000 there have been typically fewer than 30 articles per year published on this topic [5]. Since 2000 however, the number of publications on multiferroic and magnetoelectric materials grew year on year and today there are thousands of articles published per year. This is best emphasized by data in figure 2, showing the cumulative number of articles published in the period 2000 to 2013. The data is extracted from Google Scholar using the keyword "multiferroic". This method of measuring the worldwide interest in a given research topic is much more accurate than WoS because it counts all written contributions, not only peer review scientific articles.

It is believed that the recent revival of research in the magneto-electric effect and multiferroics is

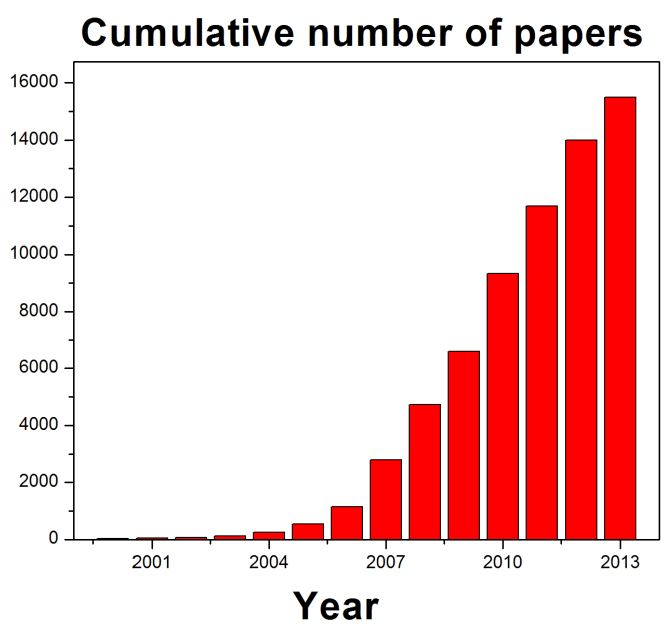

Figure 2. Cumulative number of published articles searched using the keyword "multiferroic" in Google Scholar. The data covers the period 2000 to 2013 and excludes patents.

due to the industrial demands for novel multifunctional materials and devices for advanced applications. Recent advances in the fabrication and discovery of new materials and thin films, including the ability to deposit nano and sub nano-scale thin film hetero-structures, also supported this revival of interest in multiferroic materials. A simple search using the keyword "multiferroic" in the Google Patent search engine yielded around 2000 results. The search refers to patent filing date (not granted) over the period $2000-2013$. Multiferroic patent search data is summarized as a cumulative plot in figure 3. Interestingly, according to figure 3, there are two major jumps in which most multiferroic patents were submitted, followed by relatively slow growth periods. The first jump occurred in $2002-2003$ and the second jump was in 2007 - 2008. Since 2008 the number of patents filed every year continued to grow, although at a slower pace.

Today multiferroic materials are recognized as a true emergent field of research and technologies. The fascinating properties of multiferroic materials make them ideal candidates for a new wave of technological applications with potential to generate billions of US dollars in revenue. By merging multiferroic materials with semiconductors and spintronic materials, these complex structures could, in future, replace the existing random access memories and computation technologies based on semiconductors.

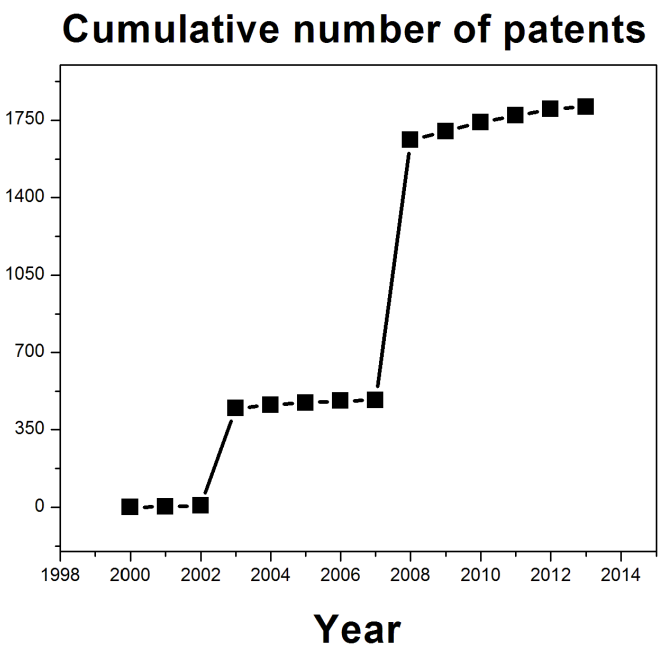

Figure 3. Cumulative number of patents by filing date searched using the keyword "multiferroic" in Google Patents. The data covers the period 2000 to 2013. 


\section{Thermodynamics of linear multiferroic materials}

Thermodynamic description of multiferroic materials is highly desirable because it offers a few clear advantages such as:

a) Thermodynamic description operates with macroscopic variables and it makes no reference to microscopic origins of the multiferroic phase or its associated couplings. This feature is particularly valuable because there is not a single universal mechanism describing the magneto-electric coupling in multiferroics, especially in the case of single-phase multiferroics.

b) Since multiferroics are classified in single-phase and composites, a microscopic theoretical model that describes both types of multiferroics is impossible. However, thermodynamic approach is indeed universally applicable, so thermodynamic relations are valid regardless whether we describe single-phase or composite multiferroics. Therefore in the following thermodynamic treatment a multiferroic material means a single entity regardless of its single-phase or composite structure.

Let's assume a multiferroic material containing both electric and magnetic phases. In addition, it is assumed that the two order phases exhibit linear coupling effects such as piezo effects, piezo coupling effects and magneto-electric coupling, while higher order coupling effects are not considered in this approach. The coupling between thermal, electric, magnetic and elastic parameters of the material can be expressed using a thermodynamic approach. Assuming the multiferroic system is subjected to a small change of strain dx, entropy dS, electric polarization $\mathrm{dP}$ and magnetization $\mathrm{dM}$, the reversible change in the internal energy of the system, dU, is given by:

$$
d U=T d S+\sigma_{i j} d x_{i j}+E_{i} d P_{i}+H_{i} d M_{i}
$$

where $\mathrm{T}$ is the temperature, $\sigma_{\mathrm{ij}}$ is the mechanical external applied stress second rank tensor, $\mathrm{E}_{\mathrm{i}}$ is the electric applied field vector, and $\mathrm{H}_{\mathrm{i}}$ is the magnetic applied field vector. In the following treatment, the tensor subscripts will be omitted for simplicity. Since in most situations we work at constant temperature and use the external applied fields and stress as independent variables, it is useful to change the thermodynamic potential from $U(S, x, P, M)$ to Gibbs free energy $\mathrm{G}(\mathrm{T}, \sigma, \mathrm{E}, \mathrm{H})$ using the Legendre transformation:

$$
G=U-T S-\sigma x-E P-H M
$$

Differentiating relation (6) and combining with (5), we obtain:

$$
d G=-S d T-x d \sigma-P d E-M d H
$$

Since $G=G(T, \sigma, E, H)$, the differential expression of the Gibbs free energy can be also written as:

$$
d G=\left(\frac{\partial G}{\partial T}\right)_{\sigma, E, H} d T+\left(\frac{\partial G}{\partial \sigma}\right)_{T, E, H} d \sigma+\left(\frac{\partial G}{\partial E}\right)_{\sigma, T, H} d E+\left(\frac{\partial G}{\partial H}\right)_{\sigma, E, T} d H
$$


Combining relations (7) and (8) we obtain, by identification, the differential expressions for entropy, stress, electric polarization and magnetization of a multiferroic system in terms of its total Gibbs free energy:

$$
\left\{\begin{array}{l}
S=-\left(\frac{\partial G}{\partial T}\right)_{\sigma, E, H} \\
x=-\left(\frac{\partial G}{\partial \sigma}\right)_{T, E, H} \\
P=-\left(\frac{\partial G}{\partial E}\right)_{\sigma, T, H} \\
M=-\left(\frac{\partial G}{\partial H}\right)_{\sigma, E, T}
\end{array}\right.
$$

Since Gibbs free energy has $(\mathrm{T}, \sigma, \mathrm{E}, \mathrm{H})$ as independent variables, the electric polarization $\mathrm{P}$, magnetization $\mathrm{M}$ and strain $\mathrm{x}$ will also have the same set of independent variables. Hence, we can differentiate them in respect with their independent variables $(\mathrm{T}, \sigma, \mathrm{E}, \mathrm{H})$, which would result in useful relations and coefficients describing coupling effects:

$$
\begin{aligned}
& d S=\left(\frac{\partial S}{\partial T}\right)_{\sigma, E, H} d T+\left(\frac{\partial S}{\partial \sigma}\right)_{T, E, H} d \sigma+\left(\frac{\partial S}{\partial E}\right)_{\sigma, T, H} d E+\left(\frac{\partial S}{\partial H}\right)_{\sigma, E, T} d H \\
& \begin{array}{cccc}
\begin{array}{c}
\text { Heat capacity } \\
\text { coefficient }
\end{array} & \begin{array}{c}
\text { Piezo-caloric } \\
\text { effect }
\end{array} & \begin{array}{c}
\text { Electro-caloric } \\
\text { effect }
\end{array} & \begin{array}{c}
\text { Magneto-caloric } \\
\text { effect }
\end{array}
\end{array} \\
& d x=\left(\frac{\partial x}{\partial T}\right)_{\sigma, E, H} d T+\left(\frac{\partial x}{\partial \sigma}\right)_{T, E, H} d \sigma+\left(\frac{\partial x}{\partial E}\right)_{\sigma, T, H} d E+\left(\frac{\partial x}{\partial H}\right)_{\sigma, E, T} d H \\
& \begin{array}{cccc}
\begin{array}{c}
\text { Thermal expansion } \\
\text { coefficient }
\end{array} & \begin{array}{c}
\text { Elastic compliance } \\
\text { coefficient }
\end{array} & \begin{array}{c}
\text { Converse piezo- } \\
\text { electric effect }
\end{array} & \begin{array}{c}
\text { Magneto-striction } \\
\text { coefficient }
\end{array}
\end{array} \\
& d M=\left(\frac{\partial M}{\partial T}\right)_{\sigma, E, H} d T+\left(\frac{\partial M}{\partial \sigma}\right)_{T, E, H} d \sigma+\left(\frac{\partial M}{\partial E}\right)_{\sigma, T, H} d E+\left(\frac{\partial M}{\partial H}\right)_{\sigma, E, T} d H \\
& \begin{array}{cccc}
\begin{array}{c}
\text { Pyro-magnetic } \\
\text { effect }
\end{array} & \begin{array}{c}
\text { Piezo-magnetic } \\
\text { effect }
\end{array} & \begin{array}{c}
\text { Magneto-electric } \\
\text { effect }
\end{array} & \begin{array}{c}
\text { Magnetic } \\
\text { susceptibility }
\end{array}
\end{array} \\
& d P=\left(\frac{\partial P}{\partial T}\right)_{\sigma, E, H} d T+\left(\frac{\partial P}{\partial \sigma}\right)_{T, E, H} d \sigma+\left(\frac{\partial P}{\partial E}\right)_{\sigma, T, H} d E+\left(\frac{\partial P}{\partial H}\right)_{\sigma, E, T} d H \\
& \begin{array}{cccc}
\begin{array}{c}
\text { Pyro-electric } \\
\text { effect }
\end{array} & \begin{array}{c}
\text { Direct piezo- } \\
\text { electric effect }
\end{array} & \begin{array}{c}
\text { Dielectric } \\
\text { susceptibility }
\end{array} & \begin{array}{c}
\text { Magneto-electric } \\
\text { effect }
\end{array}
\end{array}
\end{aligned}
$$

Each partial derivative in equations (10) - (13) represents, in the linear approximation, a physical effect as indicated below each term defining a coupling coefficient. These coefficients are called "compliances" and they provide a measure of the coupling between fields. In the case of isothermal $(\mathrm{dT}=0)$ and adiabatic $(\mathrm{dS}=0)$ piezo-multiferroic systems, 
the equations (11), (12) and (13) can be re-written in integral form and linear approximation as:

$$
\left\{\begin{array}{l}
x=s \sigma+d^{e} E+d^{m} H \\
P=d^{e, c} \sigma+\chi^{e} E+\alpha^{m} H \\
M=d^{m, c} \sigma+\alpha^{e} E+\chi^{m} H
\end{array}\right.
$$

where tensor suffixes have been intentionally neglected, superscripts $e$ and $m$ refer to electric and magnetic phase, respectively, $s$ is the elastic compliance (fourth rank tensor), $d^{e, m}$ and $d^{e, m, c}$ are the direct and converse piezo-electric and piezo-magnetic coefficients (third rank tensors), $\alpha^{e, m}$ is the magneto-electric coupling coefficient (second rank tensor) and $\chi^{e, m}$ is the dielectric or magnetic susceptibility (second rank tensor). Equations (14) are called constitutive linear equations of state of the multiferroic system. Although not expressed in tensor notations, the compliances in (14), as well as the variables are tensors of various ranks, as resulted from the following partial derivatives in (11) - (13):

$$
\begin{aligned}
& s_{i j k l}=\left(\frac{\partial x_{i j}}{\partial \sigma_{k l}}\right)_{T, E, H} ; d_{k i j}^{e}=\left(\frac{\partial P_{k}}{\partial \sigma_{i j}}\right)_{T, E, H}=d_{i j k}^{e, c}=\left(\frac{\partial x_{i j}}{\partial E_{k}}\right)_{\sigma, T, H} ; \chi_{i j}^{e}=\left(\frac{\partial P_{i}}{\partial E_{j}}\right)_{\sigma, T, H} \\
& \alpha_{i j}^{e}=\left(\frac{\partial M_{i}}{\partial E_{j}}\right)_{T, \sigma, H}=\alpha_{i j}{ }^{m}=\left(\frac{\partial P_{i}}{\partial H_{j}}\right)_{T, \sigma, E} ; d_{k i j}{ }^{m}=\left(\frac{\partial M_{k}}{\partial \sigma_{i j}}\right)_{T, E, H}=d_{i j k}{ }^{m, c}=\left(\frac{\partial x_{i j}}{\partial H_{k}}\right)_{\sigma, T, E} ; \\
& \chi_{i j}{ }^{m}=\left(\frac{\partial M_{i}}{\partial H_{j}}\right)_{\sigma, T, E}
\end{aligned}
$$

\section{Multiferroic materials}

While the piezo-ferro coupling is well understood, the physical mechanisms leading to the occurrence of the magneto-electric coupling effect in multiferroic solids are not so straightforward. Maxwell has first demonstrated the link between electricity and magnetism by introducing the well-known differential classical Maxwell equations. Maxwell equations provide the mathematical relationship between the time derivatives of electric and magnetic fields to their spatial gradients, so they refer strictly to time varying electric and magnetic fields (i.e. electromagnetic waves). The natural question one would ask is: What about static magnetic and electric fields in solids? Is there a possible coupling mechanism between the electricity and magnetism in solids? Thermodynamic relations predict such a coupling, as shown in the previous section. Also already mentioned, Pierre Curie was the first to propose a linear magneto-electric coupling in 1894 based on crystal symmetry considerations [13]. Multiferroic properties are determined by the symmetry because the primary ferroic constituents of the multiferroic compounds can be characterized by their behavior when subjected to space and time inversion. A time reversal symmetry operation would leave the electric polarization of the crystal invariant while changing the sign of the magnetization. Similarly, a spatial symmetry operation would leave the magnetization invariant, but it will reverse the electric polarization. Therefore, magnetic materials break the time inversion symmetry, while electrically ordered dielectrics break the spatial inversion symmetry. Hence, 
a multiferroic crystal consisting of both electric and magnetic order states would have to break both time and spatial inversion symmetry. In order to understand this symmetry violation requirement, it is best to re-examine the microscopic origins of the magnetic and electric phenomena. It is well known that magnetism or magnetic phenomena originate from cooperative interaction of individual entities called magnetic moments. The orbital motion of an electron inside the atom generates the most elementary magnetic moment. The magnetic dipole moment $\mu^{\mathrm{m}}$ associated with a loop of electric current $\mathrm{I}$ and area $\mathrm{A}$ is by definition given by:

$\mu^{m}=I \cdot A=\frac{d Q}{d t} \cdot A$

Applying a time inversion symmetry operation $t \rightarrow-t$, results in reversal of the magnetic moment, while a spatial inversion leaves the magnetic moment invariant (figure 4):

$$
\mu^{m \prime}=-\frac{d Q}{d t} \cdot A=-\mu^{m}
$$

We now recall that the microscopic origin of polar effects in dielectric materials is related to the collective interaction of physical entities called electric dipoles. Giving two point charges $+q$ and $-q$ separated by a distance $r$, the electric dipole $\mu^{\mathrm{e}}$ is defined as:

$$
\mu^{e}=q \cdot r
$$

Applying a spatial inversion $r \rightarrow-r$ to relation (18), we obtain a reversal of the electric dipole moment, while a time inversion leaves the electric dipole invariant (figure 4):

$$
\mu^{e \prime}=-q \cdot r=-\mu^{e}
$$

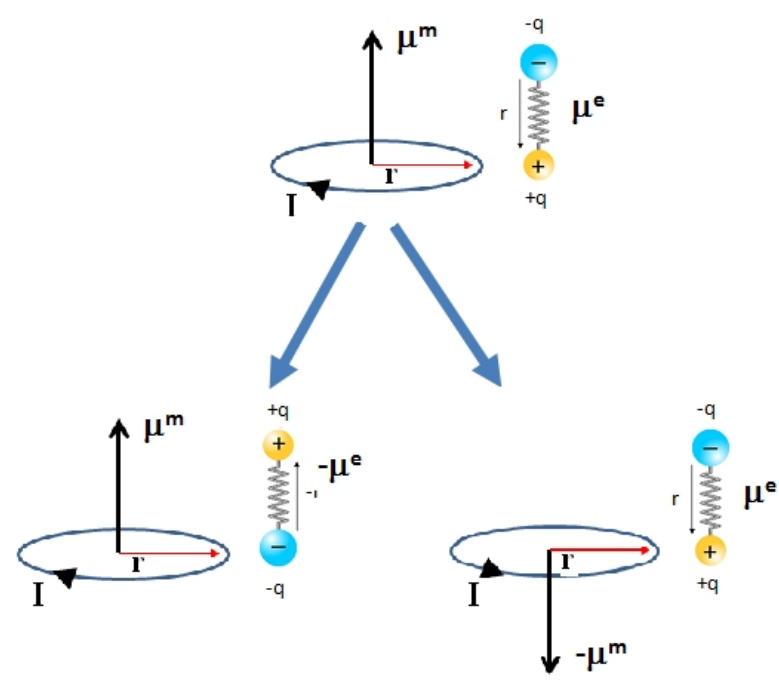

Spatial inversion symmetry Time inversion symmetry

Figure 4. Diagram of the time and spatial symmetry operation on a magnetic moment and an electric dipole moment.

Therefore multiferroics require simultaneous violation of space and time inversion symmetry.

\subsection{Single-phase multiferroic materials}

Single-phase multiferroic materials are defined as homogenous compounds and chemically isotropic, in which electric and magnetic order states coexist at any point or given location within the material. Elastic / piezo-elastic properties may also be displayed in single-phase multiferroics, although this is not a strict requirement. However, the occurrence of magnetic and electric order states in the same phase requires strict symmetry conditions. Crystals with electric order phase permitting a spontaneous electric polarization must be invariant under time reversal symmetry and magnetic ordered crystals with spontaneous magnetization must be invariant under space inversion symmetry operation. The total number of possible crystal 
structures is 122 , as given by the Shubnikov point groups [20]. Out of all 122 possible point groups, there are 31 crystallographic point groups permitting electric phase and 31- point groups allowing magnetic phase. Shuvalov and Belov showed that 13 crystallographic point groups are found in both sets and allow electric and magnetic order to coexist in the same phase (i.e. multiferroic crystals) [21,22]. The 13 crystallographic point groups allowing multiferroic phase are: 1, 2, 2', m, m', 3, 3m', 4, 4m'm', m'm2', m'm'2', 6 and 6m'm'. The multiferroic point groups are tabulated in [17, 23]. For a more recent review of symmetry aspects in ferroic and multiferroic materials, the reader is encouraged to study the article published by Schmid [24].

Table 1 shows some of the most well-known single-phase multiferroic compounds. Since one of the ferroic components is the electrically ordered phase, which requires dielectric materials, unsurprisingly all single-phase multiferroic materials are dielectric oxides. However, single-phase multiferroics rarely occur in nature and they are also very difficult to prepare artificially. The reasons behind the scarcity of single-phase multiferroics have been analyzed by Hill in an excellent article [25]. Since only 13-point groups out of a total of 122 possible crystallographic point groups accept multiferroic state, the generally accepted theory of the lack of single-phase multiferroics was linked to symmetry restrictions. However, Hill argues in her article that transition metal d-electrons, essential for magnetic phenomena, reduce the tendency for off-center ferroelectric distortion.

Single-phase multiferroics are further classified into two types: Type-I and Type-II multiferroics. These two classes are mainly related to their different physical mechanisms of electric polarization.

Type-I single-phase multiferroics are materials in which magnetic and electric phases coexist within the same compound, but they have different microscopic origins and are broadly independent of each other.

Type-II single-phase multiferroics are materials in which ferroelectric order is induced by magnetism, which in turn suggests a very strong magneto-electric coupling.

The classification of single-phase multiferroic materials is well documented in an excellent review article published by Khomskii [26].

\subsection{Multiferroic composites}

Although a substantial number of single-phase multiferroic compounds have been widely investigated, not a single material has been discovered to display large and technologically useful magneto-electric coupling at room temperature. Despite their fascinating physics and properties, the lack of single-phase multiferroics operating at room temperature, coupled with their weak magneto-electric coupling has made single-phase multiferroics uninteresting and unattractive for applications. This has resulted in a concentrated effort of the academic and research communities to take innovative approaches and to develop alternative multiferroic materials. An elegant solution to single phase multiferroics dilemma was the development of multiferroic composite materials.

Multiferroic composites are defined as compounds in which electric, magnetic and piezo order states coexist within the material, but unlike single-phase multiferroics, which are homogenous compounds and chemically isotropic, the composite multiferroics are characterized by the fact that the order phases are physically separated from each other within the multiferroic composite material. The genial idea of fabricating multiferroic composites offered substantial advantages including greater flexibility in designing the materials for specific applications and optimization of the magneto-electric coupling. Indeed, magneto- 
electric effects measured in typical composite multiferroics are a few orders of magnitude larger than those of single-phase multiferroics and most composite multiferroics operate at or above room temperature.

Table 1. Typical examples of single-phase and composite multiferroic materials $(\mathrm{PZT}=$ $\left.\mathrm{PbZr}_{1-\mathrm{x}} \mathrm{Ti}_{\mathrm{x}} \mathrm{O}_{3}\right)$.

\begin{tabular}{|c|c|c|c|}
\hline Composite Multiferroics & Ref. & Single-Phase Multiferroics & Ref. \\
\hline $\mathrm{CoFe}_{2} \mathrm{O}_{4}$ in $\mathrm{BaTiO}_{3}$ matrix & [27] & $\mathrm{Cr}_{2} \mathrm{O}_{3}$ & [41] \\
\hline $\mathrm{BaTiO}_{3}-\mathrm{CoFe}_{2} \mathrm{O}_{4}$ composite fiber & [28] & $\mathrm{Cr}_{3} \mathrm{~B}_{7} \mathrm{O}_{13} \mathrm{Cl}$ & [42] \\
\hline $\mathrm{Tb}_{1-\mathrm{x}} \mathrm{Dy}_{\mathrm{x}} \mathrm{Fe}_{2}-\mathrm{PZT}$ in polymer matrix & [29] & $\mathrm{GaFeO}_{3}$ & [43] \\
\hline $\mathrm{Tb}_{1-\mathrm{x}} \mathrm{Dy}_{\mathrm{x}} \mathrm{Fe}_{2} /$ PZT laminated layers & [30] & $\mathrm{LiCoPO}_{4}$ & [44] \\
\hline $\mathrm{Tb}_{1-\mathrm{x}} \mathrm{Dy}_{\mathrm{x}} \mathrm{Fe}_{2} / \mathrm{PZT} / \mathrm{Tb}_{1-\mathrm{x}} \mathrm{Dy}_{\mathrm{x}} \mathrm{Fe}_{2}$ laminates & [30] & $\mathrm{NdCrTiO}_{5}$ & [45] \\
\hline $\mathrm{La}_{0.7} \mathrm{Sr}_{0.3} \mathrm{MnO}_{3} /$ PZT laminated layers & [31] & $\mathrm{TbPO}_{4}$ & [46] \\
\hline $\mathrm{La}_{0.7} \mathrm{Sr}_{0.3} \mathrm{MnO}_{3} / \mathrm{BaTiO}_{3}$ laminated layers & [32] & YIG & [47] \\
\hline CoPd / PZT laminated layers & [33] & $\mathrm{BiFeO}_{3}$ & [48] \\
\hline $\mathrm{NiFe}_{2} \mathrm{O}_{4} / \mathrm{PZT}$ laminated layers & [34] & $\mathrm{BiMnO}_{3}$ & [48] \\
\hline $\mathrm{BaTiO}_{3} / \mathrm{Fe}$ laminated layers & [31] & $\mathrm{CdCr}_{2} \mathrm{~S}_{4}$ & [49] \\
\hline $\mathrm{CoFeV}$ / PZT / CoFeV laminated layers & [35] & $\mathrm{YMnO}_{3}$ & {$[50]$} \\
\hline $\mathrm{Ni}_{0.8} \mathrm{Zn}_{0.2} \mathrm{Fe}_{2} \mathrm{O}_{4} / \mathrm{PZT}$ laminated layers & [36] & $\mathrm{HoMnO}_{3}$ & [51] \\
\hline $\mathrm{Co}_{0.6} \mathrm{Zn}_{0.4} \mathrm{Mn}_{2} \mathrm{O}_{4} / \mathrm{PZT}$ laminated layers & [36] & $\mathrm{TbMnO}_{3}$ & [52] \\
\hline $\mathrm{SrAl}_{\mathrm{x}} \mathrm{Fe}_{12-\mathrm{x}} \mathrm{O}_{19} / \mathrm{PZT}$ laminated layers & [37] & $\mathrm{TbMn}_{2} \mathrm{O}_{5}$ & [53] \\
\hline FeBSiC / PZT laminated layers & [38] & $\mathrm{BaMnF}_{4}$ & [54] \\
\hline Ni / PZT / Ni laminated layers & [39] & $\mathrm{Ba}_{0.5} \mathrm{Sr}_{1.5} \mathrm{Zn}_{2} \mathrm{Fe}_{12} \mathrm{O}_{22}$ & [55] \\
\hline AlN / FeCoSiB bi-layers & [40] & $\mathrm{PbFeTiO}_{3}$ & [56] \\
\hline
\end{tabular}

Since the ferroic phases are fully separated within the composite, the requirement for "full dielectric" is no longer applicable. In the case of composites, the electric order phase is obviously a dielectric insulator, but the magnetic phase can be dielectric insulator or metallic conductor. This powerful feature increases substantially the versatility in fabrication of composite multiferroics, because a wider range of constituent materials are available.

Moreover, the limitations of single-phase multiferroics related to simultaneous spatial and time reversal symmetry breaking are completely eliminated in the case of composite multiferroics, as the symmetry condition is automatically fulfilled for any combination of materials in any possible geometry. Figure 5 shows a few possible geometries of mixing magnetic and electric phases in a multiferroic composite. More details, possible geometries and classifications of composite multiferroics can be found in these excellent review articles $[8,10,57,58]$, including the well-established Newnham multiferroic composites classification [59]. A closer inspection of figure 5 reveals two particular composite multiferroic structures that offer great promises for technological applications, namely laminated bi-layer and multilayer multiferroic composites. These structures can be fabricated as bulk, but most importantly as thin films [60].

The ability to fabricate laminated thin film multiferroic composites is a great advantage as this makes them susceptible to nano-fabrication using nano-lithography and thin film growth. Today, thin film technologies allow the precise control of the film thickness to sub nanometer level, as well as precise control of composition, stoechiometry, crystalline structure and grain size $[61,62]$. 


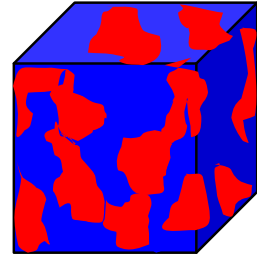

A)

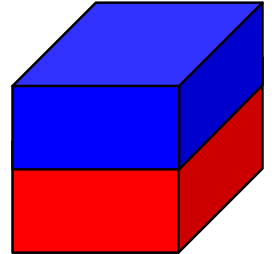

B)

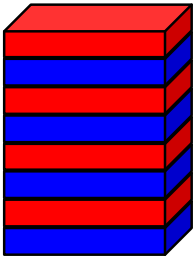

C)

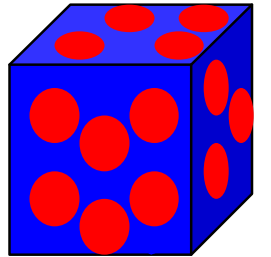

D)

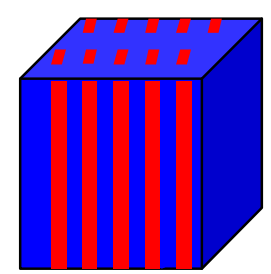

E)

Electric phase

Magnetic phase

Figure 5. Diagrams of possible multiferroic composite structures. A) Homogeneous mixture of electric and magnetic phases; B) Laminated bi-layer structure; C) Laminated multi-layer structure; D) Composite made of particles mixed in a matrix; E) Fiber multiferroic composite.

Such powerful features, combined with lithography, offer unprecedented opportunities in fabrication of novel multiferroic composite structures at nano-scale. This in turn brings a new dimension to the field of multiferroics, as nano-fabrication and thin film platforms could potentially facilitate their integration with electronic circuits, spintronic devices, data storage and silicon technologies. Moreover, it is expected that fabricating nanoscale composite multiferroics could lead to the discovery of novel effects because at such reduced length scales classical behavior of materials breaks down and interesting quantum effects become dominant.

Unlike single-phase multiferroics, in which a direct magneto-electric coupling occurs, composite multiferroics are characterized by an indirect magneto-electric coupling [11]. In the case of indirect magneto-electric effect, the application of magnetic or electric fields does not affect directly the electric polarization or magnetization, respectively. Instead, they are modified indirectly via a strain mediated magneto-electric coupling.

In other words, the magnetic and electric phases are coupled indirectly via elastic interactions (see figure 1). The applied electric or magnetic field results in a piezo-strain induced upon the order phase corresponding to the activation field and this piezo-strain is then transmitted to the other latent order phase in the multiferroic compound resulting in a variation of its order parameter through the converse piezo-effect.

Van Suchtelen first proposed in 1972 the idea of strain-mediated coupling between two ferroic phases [63]. Soon after his proposal, scientists at Philips Laboratory reported large experimental magneto-electric coupling in multiferroic composites [64-66]. Further developments were published later in 1990s when particulate ceramic composites of magnetic ferrites and ferroelectric $\mathrm{BaTiO}_{3}$ and / or PZT were successfully prepared [67-69].

The re-emergence of multiferroic materials coincided with the publication of giant magnetoelectric coupling in the early 2000s. Using giant magnetostrictive alloys such as $\mathrm{Tb}_{1-\mathrm{x}} \mathrm{Dy} \mathrm{y}_{\mathrm{x}} \mathrm{Fe}_{2}$ (Terfenol - D), combined with $\mathrm{PbZr}_{1-\mathrm{x}} \mathrm{Ti}_{\mathrm{x}} \mathrm{O}_{3}$ (PZT) piezoelectric ceramics or piezoelectric polymers such as poly(vinylidene fluoride-trifluorethylene) copolymer (PVDF-TrFE), composite multiferroics with very large magneto-electric coupling at room temperature have been reported [30,70-78]. Most recent studies in multiferroic composites reported truly remarkable magneto-electric coupling coefficients of a few Volts / cm.Oe, which when translated into field sensitivity are close to the most sensitive magnetic field detectors including SQUID and MFM [79-82]. 


\section{Possible applications of multiferroics}

\section{1 ac / dc magnetic field sensors}

Magnetic sensors are devices that can detect, without physical contact, the creation or variation in magnetic fields of the surrounding environment and from these changes they derive information on the properties of the magnetic field itself, as well as indirect properties of other entities such as: direction, presence, rotation, angle, speed, electrical currents, temperature, and so on.

The output signal of magnetic sensors requires some signal processing for translation into the desired parameter. Magnetic field sensors are widely utilized in a range of applications with huge impact on our society. Examples of such applications are: magnetic field sensors for scientific measurements and metrologies, microscopy imaging, magnetic field mapping of Earth's field, other cosmological entities and geo-positioning, detection of metals and weapons, security systems, magnetic recording readers for magnetic data storage, biomedical applications, medical imaging, positioning and speed measurement, automotive applications, to name a few.

According to a new market research report (www.marketsandmarkets.com), the demand for magnetic field sensors in the year 2010 was 3.67 billion units with a total market value of 1.1 billion US dollars. The magnetic field sensors market is expected to reach 7.14 billion units in 2016, with a total value of 2.0 billion US dollars. The main driver of growth in the magnetic sensors market is the increased demand from the automotive, industrial and consumer electronics markets. In the case of automotive industry, magnetic sensors are utilized in at least 10 applications [http://memsblog.wordpress.com/2010/04/05/isupplisilicon-magnetic-sensors-head-for-big-time/]. The most common types of magnetic field sensors are summarized in Table 2, together with their physical operating principle.

Multiferroic materials and devices can be used to fabricate magnetic field sensors by utilizing the magnetically induced magneto-electric effect. This is mathematically described by the magneto-electric voltage coupling coefficient $\left(\alpha_{\mathrm{H}}{ }^{\mathrm{V}}\right)$, which gives a relationship between the voltage induced on the electrodes of a multiferroic device and the amplitude of an externally applied magnetic field - i.e. the field to be sensed (see relation 4). Integrating relation 4 gives:

$V=\alpha_{H}{ }^{V} \cdot H \cdot t$

where $t$ is the thickness of the ferroelectric layer in the case of laminates, or the thickness of the sample in the case of a single-phase composite, $\mathrm{H}$ is the amplitude of the magnetic field under measurement and $\mathrm{V}$ is the voltage response of the multiferroic sensor.

Relation (20) indicates that the voltage response of a multiferroic sensor varies linearly with the amplitude of the applied magnetic field. A linear response is a critical requirement in order to operate as a sensor. However, the measured field $\mathrm{H}$ in this case is in fact an ac magnetic field, so almost all magnetic field sensing applications based on multiferroics are related to the detection of ac signals [30,40,70-82]. ac detection using multiferoics requires the application of a dc magnetic field bias. The dc bias field couples with the ac magnetic field to produce a pseudo-piezomagnetic linear response, which in turn modulates the electrical response of the multiferroic sensor. Relation (20) is applicable to single-phase as well as composite multiferroics. However, as seen in (20), the voltage output also depends on the strength of the magneto-electric coupling coefficient. Since single-phase mutiferroics show very small magneto-electric coupling effects and usually require cryogenic temperatures, most of the magnetic sensor applications are based on composite multiferroic 
structures, in which large strain mediated magneto-electric effects occur at room temperature. These are usually bi-layer or tri-layer composite multiferroic structures with various geometries. Obviously, for magnetic sensing applications, the strain mediated magnetoelectric coupling is magnetically induced and intimately related to the magneto-strictive properties of the magnetic phase.

Table 2. Different types of magnetic field sensors listed by their operating principle and best estimates of their approximate detection limits.

\begin{tabular}{|cccc|}
\hline $\begin{array}{c}\text { Magnetic field } \\
\text { sensor }\end{array}$ & Operating principle & Special requirements & $\begin{array}{c}\text { Magnetic field } \\
\text { detection limit }\end{array}$ \\
\hline Inductive coil & Faraday effect & ac magnetic fields only & $\sim 10^{-4} \mathrm{~T}$ \\
\hline Flux gate & Magnetic induction & Large size & $\sim 10^{-8} \mathrm{~T}$ \\
\hline Magneto-optic & $\begin{array}{c}\text { Kerr / Faraday polarization } \\
\text { rotation }\end{array}$ & Optical alignment & $\sim 10^{-12} \mathrm{~T}$ \\
\hline SQUID & $\begin{array}{c}\text { Quantum interference } \\
\text { Josephson effect }\end{array}$ & Cryogenic cooling & $\sim 10^{-15} \mathrm{~T}$ \\
\hline Hall sensor & Hall effect/Lorentz force & - & $\sim 10^{-5} \mathrm{~T}$ \\
\hline AMR & $\begin{array}{c}\text { Anisotropic magneto- } \\
\text { resistance }\end{array}$ & dc biasing & $\sim 10^{-3} \mathrm{~T}$ \\
\hline GMR & $\begin{array}{c}\text { Spin dependent interface } \\
\text { scattering }\end{array}$ & - & $\sim 10^{-3} \mathrm{~T}$ \\
\hline TMR & $\begin{array}{c}\text { Spin dependent quantum } \\
\text { tunneling }\end{array}$ & - & $\sim 10^{-3} \mathrm{~T}$ \\
\hline CMR & $\begin{array}{c}\text { Double exchange and } \\
\text { semiconductor-to-metallic } \\
\text { transition }\end{array}$ & $\begin{array}{c}\text { Highly temperature } \\
\text { dependence }\end{array}$ & $\sim 10^{-2} \mathrm{~T}$ \\
\hline Rubidium gas & Spin-exchange relaxation & Cryogenic cooling & $\sim 10^{-14} \mathrm{~T}$ \\
\hline Multiferroic & Magneto-electric coupling & dc biasing & $\sim 10^{-12} \mathrm{~T}$ \\
\hline & $\quad$ & & \\
\hline
\end{tabular}

It is well known that magnetic strain, as a function of the dc-applied magnetic field, is highly non-linear. At zero applied magnetic fields, the strain is zero. Increasing the applied magnetic field the strain increases rapidly and, at a given field called the saturation field, the magnetic strain becomes saturated. Beyond this point, further increases in the magnetic applied field have no effect on the sample's strain. This saturation magnetic field roughly coincides with the saturation magnetization of the sample on the magnetic hysteresis loop. The non-linearity of the magnetic strain is transferred to the piezomagnetic or magneto-strictive coefficient (defined as the derivative of the strain in respect with the applied field) and this in turn is transferred to the magneto-electric coupling coefficient. A typical voltage response of a multiferroic composite sensor

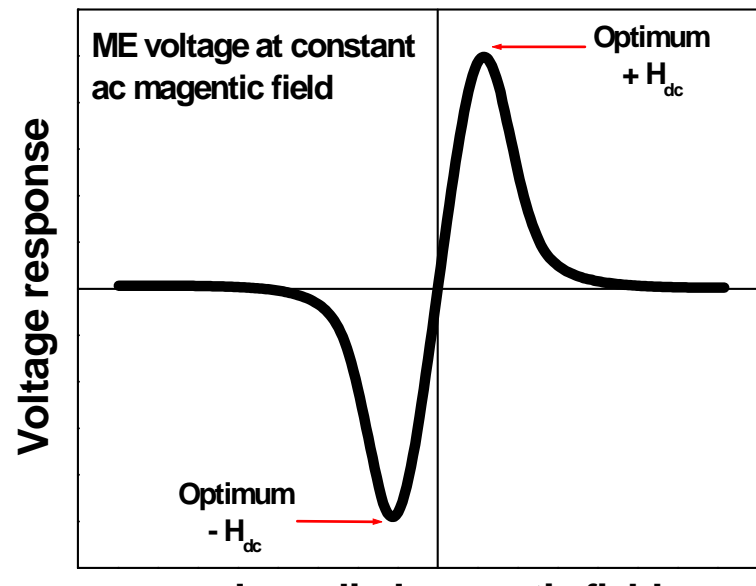

dc applied magnetic field

Figure 6. Typical response of a composite multiferroic to a dc applied magnetic field at constant ac magnetic field amplitude and frequency. to applied dc magnetic fields at constant ac magnetic field amplitude and frequency is shown in figure 6. 
The magneto-electric voltage response has a maximum at a specific $+/-$ dc magnetic bias field. This optimum dc bias field corresponds to the maximum piezo-magnetic coefficient, which in turn corresponds to the point of largest gradient in the strain - field curve. The dcresponse of composite multiferroics implies that they are not suitable for dc-magnetic field sensors. However, they can operate linearly as ac-magnetic field sensors, with the best voltage response obtained when the sensors are magnetically biased by a suitable dc magnetic field.

Therefore, not surprisingly most applications of multiferroic composites are related to detection of ac magnetic fields. Experimental and theoretical results indicate promising qualities of multiferroic sensors with magnetic field detection sensitivities varying from $10^{-13}$ $<\mathrm{H}_{\mathrm{ac}}<10^{-3} \mathrm{~T}$ [30, 40, 70-81], especially when driven at electromechanical resonance frequency [77, 82, 83]. For more details on multiferroic composite sensors, the following excellent review articles are strongly recommended to the reader $[6,8,10,57]$.

It is important to mention that the angle between the dc bias magnetic field vector and ac excitation field is critical for the operation of the multiferroic sensor. Studies of the magnetoelectric coupling coefficient as a function of this angle have indicated that the maximum coupling effect is achieved when the fields are parallel to each other, while the effect is zero for the transversal case [84]. In fact coupling measured at intermediate angles between $0^{0}$ and $180^{\circ}$ shows almost a linear variation of the sensor's response [84]. This indicates the possibility of utilizing multiferroic sensors for direction detection / compass applications. Indeed, Lage et al. have recently reported the realization of a vector field magnetometer based on two orthogonal self-biased multiferroic composite sensors that can be used for the detection of 2D magnetic field vectors and, in principle, it can be expanded to 3D magnetic field vector mapping [85].

In terms of dc magnetic field detection, the linearity condition in dc-magnetic field must be fulfilled. Examining figure 6, there is a range of dc-magnetic fields where the response is indeed linear. This will depend on the geometry and constituent materials of the sensor, but essentially would allow detection of small dc magnetic fields with sensitivity limit of about $10^{-7} \mathrm{~T}$, as already reported in [86].

Other mechanism in which a linear dc magnetic field response is possible, is when a composite multiferroic displays linear behavior in $\mathrm{M}(\mathrm{H})$ curves (i.e. no hysteresis loop) or a linear response in strain - field curves. Such conditions can be fulfilled by a multiferroic containing a paramagnetic phase or a ferromagnetic phase with strong perpendicular magnetic anisotropy. The concept of multiferroic composite magnetic field sensor for dual detection of dc / ac magnetic fields has been indeed demonstrated experimentally [37]. In this paper it has been shown experimentally that a linear magneto-electric response can be achieved for both ac and dc applied magnetic fields by using multiferroic $\operatorname{SrAl}_{\mathrm{x}} \mathrm{Fe}_{12-\mathrm{x}} \mathrm{O}_{19}$ (hexaferrite) / $\mathrm{PbZrTiO}_{3}$ (PZT) laminates with strong perpendicular magnetic anisotropy. These kind of structures can therefore operate in dual mode as both ac and dc sensors, with linear dc response over the $-2 \mathrm{kOe}<\mathrm{H}_{\mathrm{dc}}<+2 \mathrm{kOe}$ field range.

\subsection{Multiferroic microwave resonators tuned electrically}

Ferromagnetic materials subjected to high frequency excitations display the so-called phenomenon of ferromagnetic resonance (FMR) [87]. The FMR is related to the fact that perturbation of the magnetization vectors or magnetic moments / spins will result in a 
relaxation of the magnetic vector via a precession motion to its new equilibrium position. Ferromagnetic resonance occurs when the frequency of the precession matches the frequency of the excitation field and this typically occurs at microwave frequencies in ferrite materials. Hence, ferromagnetic resonance devices based on soft ferrite materials are utilized in microwave signal processing devices such as resonators, bandpass / band-stop filters and phase shifters operating at $1-70 \mathrm{GHz}$.

Such devices do require a dc magnetic bias field to operate and to achieve frequency tuning. However, magnetic field tuning has disadvantages such as slow operation, large noise level, large power consumption and limited miniaturization. Radar and communication technologies require rapid switching from one operating frequency to another with minimal power. By replacing the ferrite materials with a multiferroic bi-layer composite,

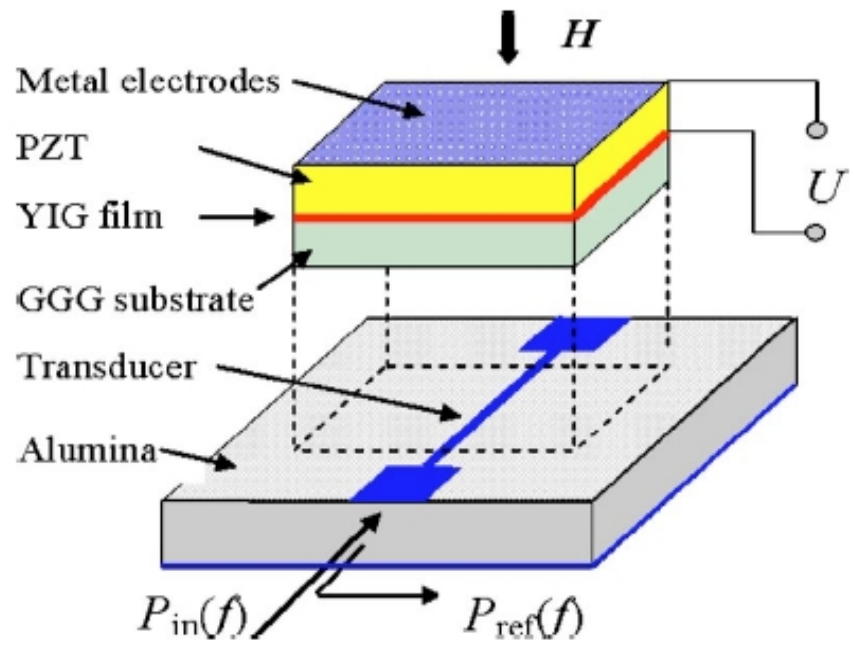

Figure 7. Diagram of the micro strip-line multiferroic resonator. Image reprinted with permission from Appl. Phys. Lett. 88, 143503 (2006) electric field tuning of FMR is possible [88]. Fetisov et al. [89] demonstrated experimentally the electric field tuning of FMR for a YIG/PZT multiferroic composite. The YIG was $15 \mu \mathrm{m}$ thick with lateral dimensions of $1 \times 2.2 \mathrm{~mm}^{2}$. The thickness of the PZT layer was $4 \mathrm{~mm}$ and its lateral dimensions $4 \times 0.5 \mathrm{~mm}^{2}$. PZT had silver electrodes on both sides and then was bonded to the YIG to form a composite multiferroic. The device consisted of a single micro strip $50 \mu \mathrm{m}$ thick and $3 \mathrm{~mm}$ long deposited onto an alumina substrate, as shown in figure 7. For a signal input of $2-10 \mathrm{GHz}, 0.1 \mathrm{~mW}$ power applied to the micro strip-line, $1.12 \mathrm{kOe} \mathrm{dc}$ in-plane magnetic bias field and $0 \mathrm{kV} / \mathrm{cm}$ electric applied field, a well-defined FMR absorption has been measured at around $5 \mathrm{GHz}$ with a linewidth of $3.4 \mathrm{MHz}$ (see figure 8).

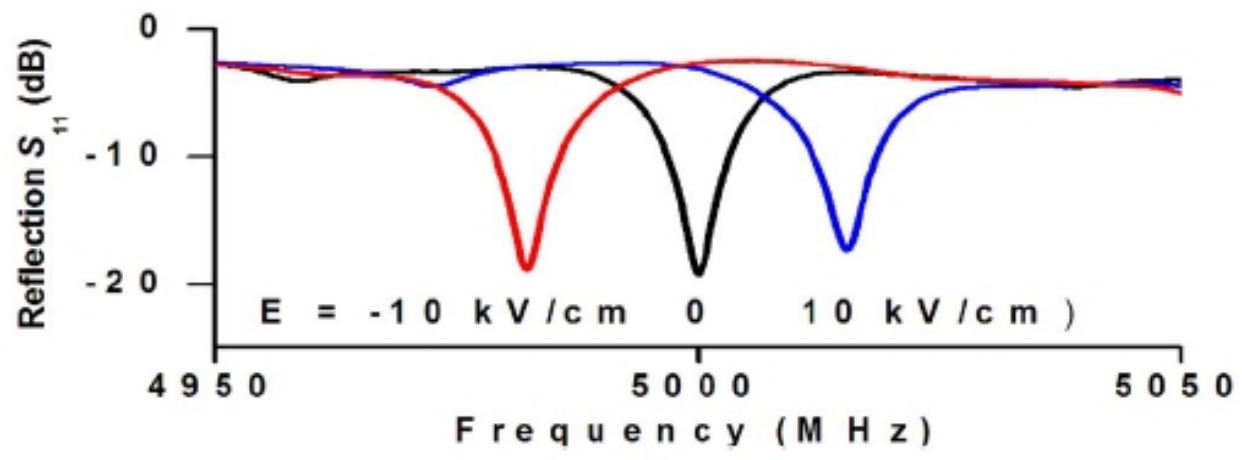

Figure 8. FMR absorption peaks of YIG measured at $1.12 \mathrm{kOe}$ in-plane dc magnetic field at $\mathrm{E}=0$ and $\mathrm{E}= \pm$ $10 \mathrm{kV} / \mathrm{cm}$. Image reprinted with permission from Appl. Phys. Lett. 88, 143503 (2006).

When an electric field $\mathrm{E}=10 \mathrm{kV} / \mathrm{cm}$ was applied, the FMR resonance shifted to a higher frequency by $16 \mathrm{MHz}$. Similarly, when a negative electric field $\mathrm{E}=-10 \mathrm{kV} / \mathrm{cm}$ was applied, the FMR peak shifted to a lower frequency by $-18 \mathrm{MHz}$. During all three measurements, the shape of the FMR response has not been altered, except its peak position. The observed shift is induced by the strain-mediated magneto-electric coupling in the multiferroic bi-layer. The 
application of the electric field has the effect of inducing a piezo-strain in the PZT, which is then transmitted to the YIG. This in turn will have the effect of changing the internal magnetization of the YIG. Therefore, the effect of the applied electric field can be viewed as the creation of an internal magnetic field, responsible for the shift in the FMR curves. Fetisov and Srinivasan estimated, for $10 \mathrm{kV} / \mathrm{cm}$ applied electric field, the induced internal magnetic field to be 5.77 Oe [89].

\subsection{Multiferroic microwave phase shifter}

Microwave phase shifters are important devices for radar applications, telecommunications and phased array antenna systems. Typical phase shifters are based on semiconductors, ferroelectrics and ferrite materials. Functionality of ferrite phase shifters is based on Faraday rotation of electromagnetic radiation in magnetized ferrites, although this is inconvenient, as it requires large applied magnetic bias fields. Another functionality mechanism of ferrite phase shifters is based on the propagation of spin waves in planar ferrites, which is very attractive due to their magnetic tunability over a wide frequency range, from $1-26 \mathrm{GHz}$.

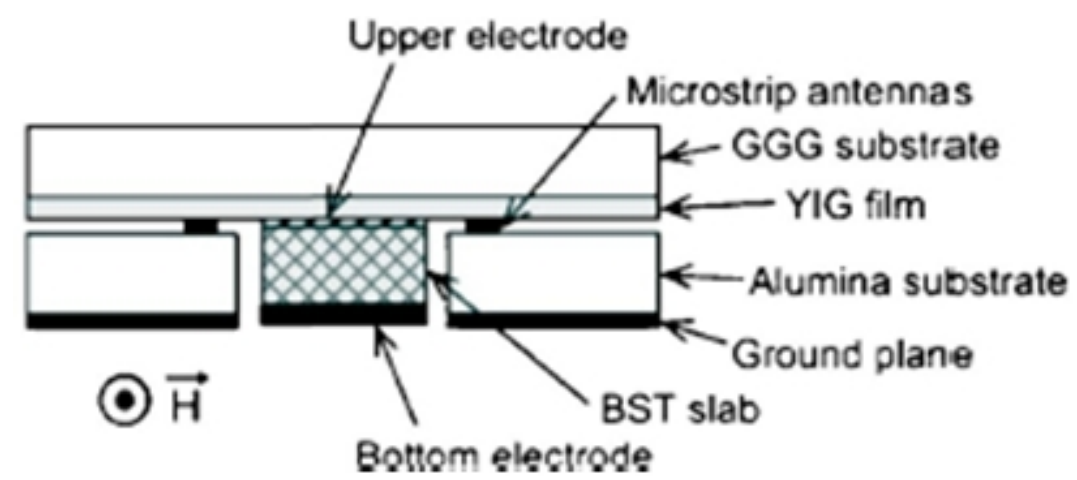

Figure 9. Diagram of the cross section of phase shifter multiferroic composite device. GGG = Gadolinium Gallium Garnet substrate; YIG = Yttrium Iron Garnet; $\mathrm{BST}=\mathrm{Ba}_{0.6} \mathrm{Sr}_{0.4} \mathrm{TiO}_{3}$; Image reprinted with permission from Appl. Phys. Letter. 90, 031913 (2007).

In contrast to magnetic ferrite phase shifters, ferroelectric phase shifters are characterized by fast electric tunability, low power consumption, but very high loss at frequencies above 5 $\mathrm{GHz}$. By combining magnetic ferrite materials with ferroelectrics it is possible to form a multiferroic composite, operating as a dual phase tunable microwave device operating well beyond $5 \mathrm{GHz}$ frequencies. Following theoretical studies [90], Ustinov et al. have reported developments of such devices [91]. Their multiferroic dual phase shifter was a bi-layer multiferroic composite consisting of a $500 \mu \mathrm{m}$ thick $\mathrm{Ba}_{0.6} \mathrm{Sr}_{0.4} \mathrm{TiO}_{3}$ ferroelectric layer and a $5.7 \mu \mathrm{m}$ thick YIG ferrite (figure 9). Lateral dimensions of the multiferroic element were $5 \times$ $10 \mathrm{~mm}$. The bottom electrode was a $\mathrm{Cu}$ layer $5 \mu \mathrm{m}$ thick, while the top electrode was a much thinner $50 \mathrm{~nm}$ Cr layer, in order to allow microwave radiation penetration to the dielectric ferroelectric. Figure 10 shows the differential phase shift for $0 \mathrm{~V}, 500 \mathrm{~V}$ and $1000 \mathrm{~V}$ applied to the ferroelectric phase, corresponding to $0,10 \mathrm{kV} / \mathrm{cm}$ and $20 \mathrm{kV} / \mathrm{cm}$ applied electric fields, respectively and a dc bias magnetic field of 1413 Oe. Similar measurements have been performed at different dc magnetic bias fields and identical electric applied fields resulting in the tuning of the performance frequency from 3.5 to $8 \mathrm{GHz}$. Electrically controlled microwave phase shifters based on stress-mediated magneto-electric coupling have also been 
reported in YIG / PZT multiferroic composites, using a device identical to the one presented in the next section [92].

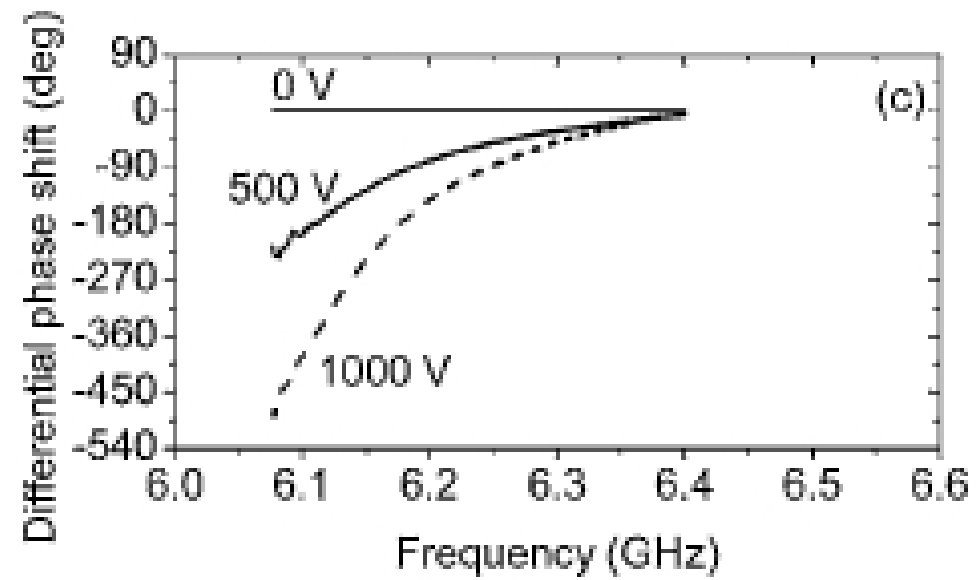

Figure 10. Electrical tuning of the differential phase shift of microwave signals measured at an applied bias magnetic field of 1413 Oe. Image reprinted with permission from Appl. Phys. Letter. 90, 031913 (2007).

\subsection{Multiferroic microwave signal delay line}

Delay lines are typically made of a ferrite thin film placed across two micro strips microwave transducers. The first micro strip transducer is connected to the input signal and the second transducer is connected to the output signal. Such devices are widely used in high frequency signal processing. The high frequency electromagnetic waves fed into the input micro strip propagate to the output micro strip via the ferrite material bridge. In this process the electromagnetic waves excite the spin waves in the ferrite material. Since the wavelength and velocity of the spin waves is almost two orders of magnitude smaller than that of the electromagnetic input signal at the same frequency, it is possible to induce a signal propagation delay as large as $500 \mathrm{~ns}$, when the propagation distance within the ferrite material is several millimeters.

Current devices rely on the fact that spin waves velocity is strongly dependent of the magnetic bias field, which allows

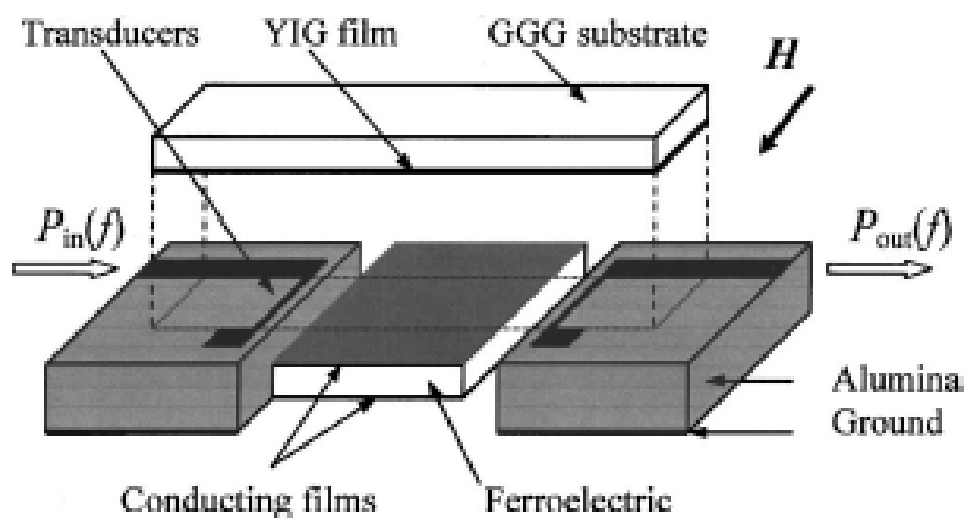

Figure 11. A microwave time delay line device based on a composite multiferroic. Image reprinted with permission from Applied Physics Letters, 87103502 (2005). delay time. While this technique is fully functional, it has a number of limitations related to the speed of tuning, power consumption associated with the magnetic bias field and size limitations prohibiting the miniaturization and integration of such devices into solid-state chips.

To solve these limitations, Fetisov and Srinivasan proposed a new design of microwave delay lines, in which they replaced the ferrite element with a bi-layer composite multiferroic with the structure ferrite/ferroelectric [93]. The materials used in their experiment were Yttrium- 
Iron-Garnet (YIG) ferrite and Lead-Magnesium-Niobate-Lead-Titanate (PMN-PT) ferroelectric (see figure 11).

The thickness of the YIG is $4.1 \mu \mathrm{m}$ and lateral dimensions $2 \times 25 \mathrm{~mm}^{2}$. The gold micro strip transducers were $50 \mu \mathrm{m}$ wide and $3 \mathrm{~mm}$ long deposited on alumina substrates via photolithography. The distance between the transducers was $8 \mathrm{~mm}$. The ferroelectric PMN-PT was $500 \mu \mathrm{m}$ thick and $4 \times 4 \mathrm{~mm}$ lateral dimensions.

The main improvement of their proposed design is the ability to tune the delay time by applying a voltage (i.e. electric field) to the multiferroic-tuning element. This is possible due to the $\mathrm{E}$ field dependence of the ferroelectric dielectric permittivity of the multiferroic element. This

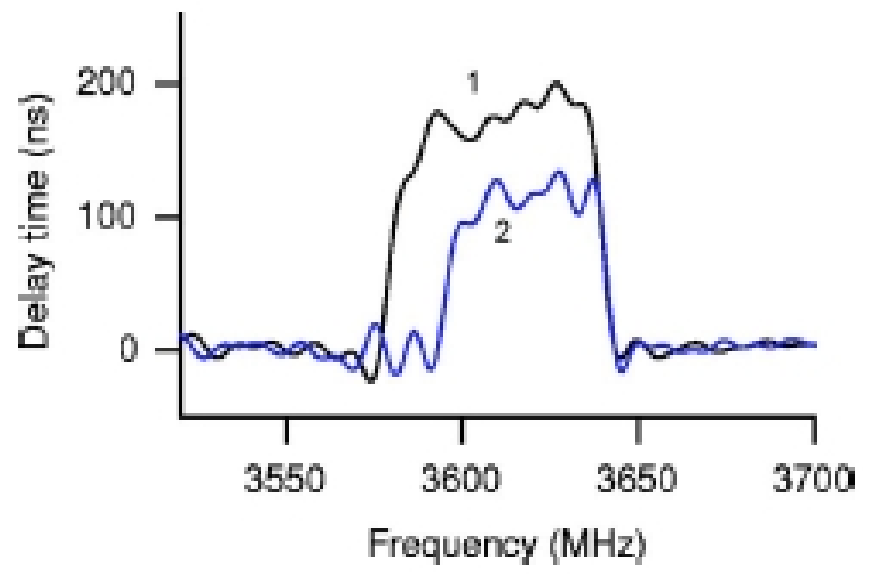
electrical tuning mechanism is fast,

Figure 12. Delay time as a function of frequency for the YIG-PMN-PT multiferroic delay line. 1) no electric field; 2) $\mathrm{E}-8 \mathrm{kV} / \mathrm{cm}$ electric field applied; Magnetic bias field was $\mathrm{H}=700$ Oe. Image reprinted with permission from Applied Physics Letters, 87103502 (2005).

power efficient and allows further device miniaturization. The authors measured a $10-25 \%$ variation in the delay time measured when the electric applied field was increased from 0 to 8 $\mathrm{kV} / \mathrm{cm}$, as evidenced in figure 12 .

\subsection{Multiferroic magnetic recording read heads}

Modern magnetic hard disk drive (HDD) technology stores digital data in tiny regions on a magnetic thin film magnetized perpendicularly up or down, called "perpendicular magnetic recording media" (PMRM). The transition from longitudinal to perpendicular recording has allowed the industry to maintain the growth path in the areal storage densities, which almost doubled every two years since the first HDD developed by IBM in 1956. After the first commercial introduction of PMRM in 2005 by Toshiba, followed by Seagate, Hitachi, Western Digital and Fujitsu in 2006, the technology has reached unprecedented areal densities of $500-700 \mathrm{~Gb} / \mathrm{in}^{2}$ in present day commercial HDDs. The digital data is retrieved from the recording medium using a magneto-resistive (MR) read head [94-98]. The MR heads operate using anisotropic magneto-resistive (AMR), giant magneto-resistance (GMR) or tunneling magneto-resistance (TMR) effects. MR effects imply a change in the internal resistance of a read sensor when interacting with the stray field from the magnetic recorded bits of a magnetic recording medium. A dc current passes through the sensor stack either current in-plane (CIP) or perpendicular to the plane (CPP) and the change in the stack resistance is translated into a read signal as a voltage amplitude change $\Delta \mathrm{V}=\mathrm{I} \times \Delta \mathrm{R}$. With the unprecedented increase in the recording densities, the read head sensor must be scaled down in order to read data effectively from smaller and smaller magnetic bits. However, reducing the size of the sensor brings a number of complications including the construction of the sensor itself becoming very challenging for sensor stacks containing minimum 15 layers with a total thickness $<20 \mathrm{~nm}$ (this is about twice the width of a data bit at $1 \mathrm{~Tb} / \mathrm{in}^{2}$ areal density). 
A possible solution is a new reader technology, which is based on the magneto-electric effect in multiferroic materials rather than one of the traditional MR effects. This technology has been first proposed in 2007 [99,100] and experimentally demonstrated in 2008 [101]. The proposed design for a multiferroic magnetic recording read head is shown in figure 13. The sensor stack is a planar structure that incorporates a tri-layer Ferromagnet (FM)/ Ferroelectric (FE)/ Ferromagnet (FM) multiferroic composite structure. This operates as an ac magnetic field detector via the magnetically induced magneto-electric effect. Essentially, as the head moves along the magnetic recording track, the field from a recorded bit provides the ac excitation field to the multiferroic read head sensor. In order to induce a strain mediated magneto-electric effect, a dc magnetic bias field is also required. The best way to bias the multiferroic composite sensor is via a self-biasing mechanism using the exchange bias effect between the FM layers with the anti-FM layers [102,103]. Other ideas of self-biased multiferroics involve hysteretic ferromagnets, but these are less robust as they rely on the demagnetization states to achieve the self-bias effect [104].

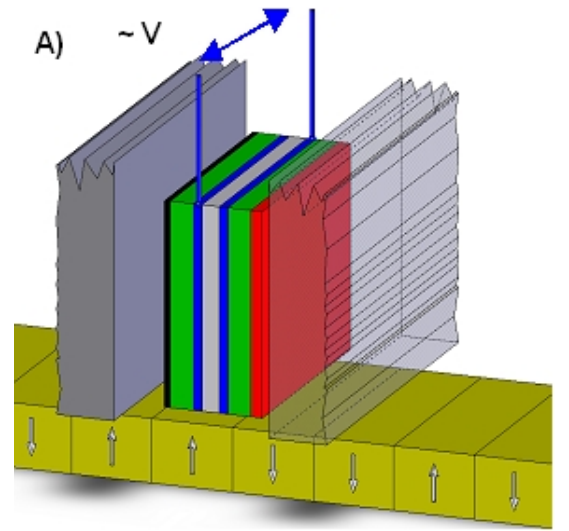

B)
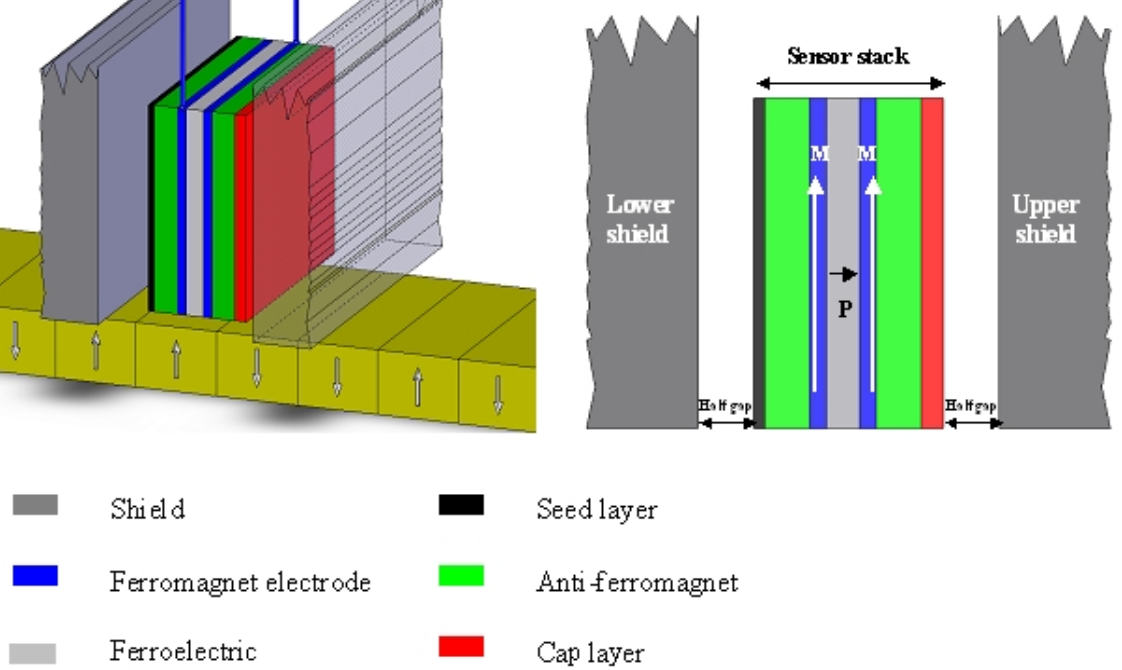

Figure 13. A) 3D diagram of the magnetic read head showing the sensor stack, half gaps, magnetic shields and a recording track; B) Cross section of the head diagram. Image reprinted with permission from J. Appl. Phys., 103, 07F506 (2008).

The use of exchange bias effect to create self-biased multiferroic thin film structures is a very novel method of designing multiferroic sensors and it has been first proposed in the same article [99]. Enno et al. confirmed experimentally the concept of self-biased multiferroics five years later [105] and currently this method is deployed to manufacture the most sensitive ac magnetic field sensors based on self-biased multiferroic composite structures [40,79-81].

In order to ensure the biasing of both FM electrodes, the proposed sensor stack must consist of 7 layers arranged as: Seed / AFM / FM / FE / FM / AFM / cap (see figure 13).

Theoretical calculations predict the open circuit voltage output of the proposed multiferroic read head to be: 


$$
V_{o c}=\frac{d_{31}{ }^{e} g_{11}{ }^{m} s_{11}{ }^{e}(1-\eta) \eta}{\varepsilon_{33} s_{11}{ }^{e}\left[\eta s_{11}{ }^{m}+(1-\eta) s_{11}{ }^{e}\right]-\left(d_{31}{ }^{e}\right)^{2}\left[(1-\eta) s_{11}{ }^{e}+\eta s_{11}{ }^{m}\left(1-\frac{\tan k a}{k a}\right)\right]} \cdot B_{1} \cdot t \cdot \frac{\tan k a}{k a}
$$

with $k=\omega \sqrt{\frac{\left(\rho_{m}(1-\eta)+\rho_{e} \eta\right)}{s_{11}{ }^{e}(1-\eta)+s_{11}{ }^{m} \eta} s_{11}{ }^{e} s_{11}{ }^{m}}$

where: $\rho_{\mathrm{m}}$ and $\rho_{\mathrm{e}}$ are the densities of the FM and FE materials, respectively and the numbers indicate the matrix components; $\eta$ is the thickness ratio defined as $\eta=t_{\mathrm{e}} / \mathrm{t}$ with $\mathrm{t}$ the total thickness of the multiferroic sensor and $t_{e}$ the thickness of the ferroelectric layer; $\omega$ is the angular frequency of the ac magnetic field; $\mathrm{d}, \mathrm{g}, \mathrm{s}, \varepsilon$ and $\beta$ are the piezoelectric constant, magnetostrictive constant, elastic compliance, permittivity and the inverse permeability tensors. Relation (21) indicates that the output voltage depends strongly on the material parameters, thickness ratio, total thickness, frequency and amplitude of the applied ac magnetic field. A full derivation of these equations can be found in ref $[99,100]$.

Assuming $1 \mathrm{~Tb} / \mathrm{in}^{2}$ areal density, numerical estimations performed for this multiferroic read head indicated, in the low frequency approximation $(\tan (\mathrm{ka}) / \mathrm{ka} \cong 1$ for $\mathrm{ka} \rightarrow 0)$, a reader output of $5.7 \mu \mathrm{V}[99,100]$. If the multiferroic read sensor operates at the electromechanical resonance frequency (i.e. $\mathrm{ka}=\pi / 2$ ), the calculated output voltage is $1.57 \mathrm{mV}$, which corresponds to a $3.14 \mathrm{mV}$ peak-to-peak signal [100].

Calculations were performed for a reader width $29 \mathrm{~nm}$, bit length $11 \mathrm{~nm}$, shield-to-shield spacing $22 \mathrm{~nm}$, half gap thickness $5 \mathrm{~nm}$, sensor stack thickness $12 \mathrm{~nm}$ and media field 100 Oe. The sensor length (e.g. stripe height - as known in the magnetic recording industry) was assumed larger than its width and thickness so that it operates in the length expansion bar, $\mathrm{L}$ $=120 \mathrm{~nm}$. The stripe height dictates the resonance frequency of the reader (i.e. operation frequency), which in the case of $120 \mathrm{~nm}$ stripe height resulted in a resonance frequency of 10.7 GHz. This can be tuned via the sensor length and it shows that a multiferroic read head operates at much higher frequencies than conventional MR readers.

The predicted advantages of such multiferroic reader technology against the existing magneto-resistive readers are multifold, including reduced power consumption, improved thermal performance as the multiferroic reader is capacitive rather than resistive, higher operation frequency and thinner reader containing maximum 7 layers, which allow magnetic recording densities of $1 \mathrm{~Tb} / \mathrm{in}^{2}$ and beyond.

\subsection{Multiferroic random access memories and multi-state memories}

Random access memory (RAM) is a key component in computer memories and consumer electronics. Depending on the technology used, RAM could be volatile or non-volatile. Volatile RAM means that computer chips store information as long as electric power is supplied to them. Once power is turned off, the information is lost. RAM memory, although volatile, offers much faster data access times than conventional non-volatile data storage. For this reason, RAM is mainly utilized to store / run the operating system code, operating software and all the associated memory operations in conjunction with the microprocessor. There are a few different types of RAM devices, but one of the most common is the dynamic random access memory (DRAM). DRAM stores each bit of data in a separate capacitor transistor (1C-1T) element within an integrated solid-state circuit. A 0 bit data state is represented by a charged capacitor state and a 1-bit state is a discharged capacitor state. These are essentially nano-scale solid-state elements and at such reduced dimensions the 
capacitors will slowly discharge in time. Hence, the information (charge on the capacitors) must be refreshed periodically, giving its name "dynamic" memory. This is in contrast to "static" random access memory (SRAM), which needs no periodic charge / data refreshing, but it has the disadvantage of being more structurally complex with $4-6$ transistors per bit element, making DRAM suitable for much larger data densities than SRAM.

In 1952, an MIT graduate student first proposed a non-volatile RAM technology based on ferroelectrics (FRAM) [106]. Replacing volatile RAM with non-volatile RAM could prevent data loss and would enable computers to start instantly, without waiting for software to boot up. Mass production of FRAM began in 1992 and today Fujitsu is the World largest manufacturer and supplier of FRAM technologies. FRAM has similar cell architecture to DRAM containing one transistor and one capacitor per bit cell (1T-1C). However, unlike the DRAM, in which the binary data is stored as charged / discharged cell's capacitor, the FRAM cell stores the binary data as remanent electric polarization states of the ferroelectric capacitor. Despite its non-volatility, FRAM is limited in applications by the need for destructive read and reset operation.

Another non-volatile RAM technology is the magneto-resistive or magnetic random access memory (MRAM) technology, which stores data bits using magnetic states and the property of magneto-resistance in multilayers. The data is encoded into a MRAM bit cell as two possible resistance states of a complex nano-scale stack of magneto-resistive multilayers. A high resistance state of the multilayers stack would correspond to a binary " 1 " and a low resistance state represents a binary " 0 ". The memory MRAM element is essentially a tunneling magneto-resistive (TMR) or giant magneto-resistive (GMR) sensor. The data read process of a given MRAM cell consists of simply interrogating the resistance state of the cell. The data write process consists in the rotation of magnetization of the sensing free layer to either a parallel or anti-parallel state relative to a magnetically pined layer in the same magneto-resistive sensor. However, changes in the magnetization state of the free layer are not inflicted by the application of an external magnetic field. They are instead generated via a magnetic field produced by carefully tuned currents passing through the bit line and word line simultaneously. This is a highly power inefficient process, which has also some thermal issues and limits dramatically the applicability of MRAM technology.

A possible solution to the issues of FRAM and MRAM is the use of multiferroic random access memory (MFRAM) technology. When magnetic order and electric polarization are simultaneously displayed, a multiferroic element could essentially combine the advantages of FRAM and MRAM to form a non-volatile random access memory with multiple memory states. Two binary states could be encoded by the ferroelectric polarization state and, by integrating the multiferroic within a magneto-resistive multilayer, two additional binary states could be encoded in the resistance state of the multilayer stack.

Figure 14 shows the schematic diagram of the proposed MFRAM technology. This consists of a multiferroic single-phase layer displaying both magnetic and electric ferro-states. This layer can be addressed electrically to rotate its polarization state while its magnetic state is pinned into a specific orientation. This could be easily achieved using the exchange bias effect for example.

The data is encoded as the two polarization states of the ferroelectric phase of the multiferroic layer, similar to the FRAM technology. A thin non-magnetic spacer layer separates the multiferroic layer from the ferromagnetic free layer. The free layer is free to rotate its magnetization parallel or anti-parallel to that of the multiferroic layer. 
When interrogated electrically, the sensor stack would display high or low resistance depending on the magnetization state of the free layer and multiferroic layer (GMR effect). If a thin dielectric layer replaces the non-magnetic spacer, the TMR effect is induced instead.

The two-resistance state encodes two memory states exactly as in the MRAM technology, except that this time two additional memory states are encoded by the electric polarization of the multiferroic layer.
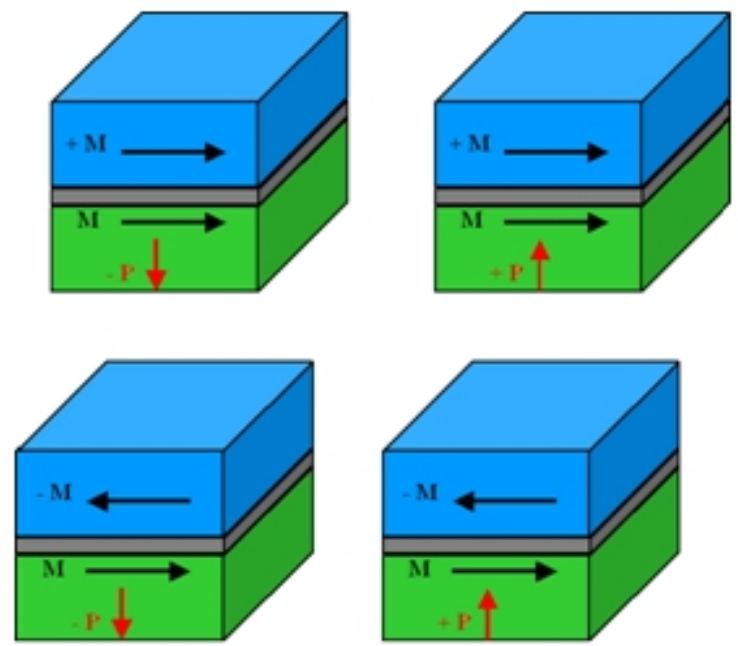

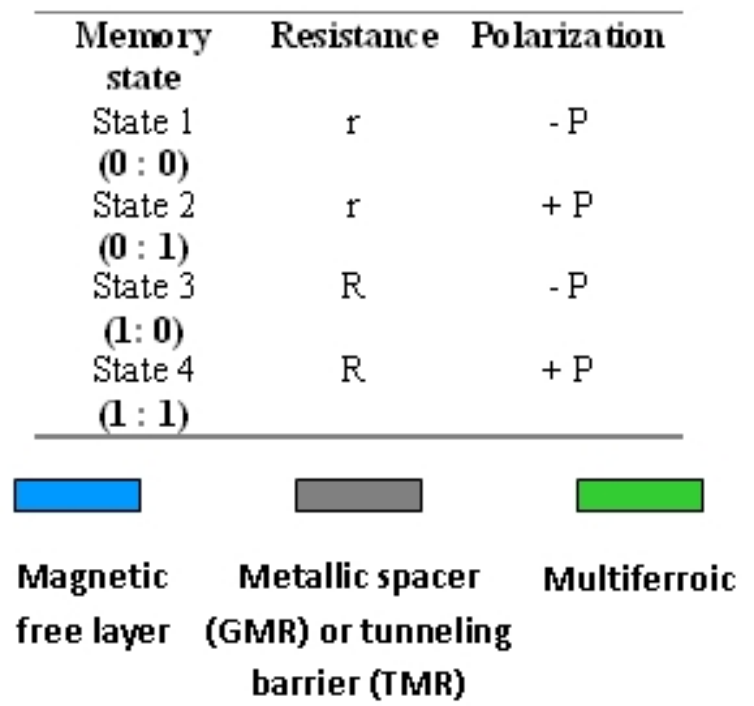

Figure 14. Schematic diagram of a 4-state MFRAM memory element based on composite multiferroics. The table shows the four possible memory states of the device.

The four possible memory states are summarized in the insert table of figure 14, where $\mathrm{r}$ means low resistance state and $\mathrm{R}$ is high resistance state. The natural question one would ask is: How the free layer rotates its magnetization in a MFRAM?

After all, the main drawback of MRAM is the inconveniences of write process in which the free layer is rotated by magnetic fields generated by a combination of bit line and word line currents. In the case of MFRAM, the well-known strain mediated magneto-electric coupling in multiferroics is utilized to achieve the magnetization rotation of the free layer. Essentially, a voltage applied to the multiferroic layer would induce a mechanical piezo-strain. This is then transferred to the free layer resulting in a change of its magnetization state. This approach to voltage control of magnetization of the free layer has been also proposed by Sun and Liu in [107] and [108]. However, in these articles the proposed MFRAM is not a 4-states memory element. As proposed here, essentially a 4-states memory element requires two write processes. Electric write process is the switching of the electric polarization of the ferroelectric phase in the multiferroic and requires saturating electric applied voltages. Magnetic / magneto-resistive write process is the rotation of the free layer magnetization, via the strain-mediated magneto-electric effect. This requires again an applied voltage, but not a saturating voltage, which means that the magnetic write process would leave the electric polarization state unaffected (no switching occurs if applied field is smaller than the coercive field). The main advantages however, is that MFRAM could operate as an all-electric read / write 4-states memory RAM, offering therefore less power consumption, more memory density and non-volatility simultaneously. Its integration would be identical to the current architecture of MRAM and FRAM, with 1T-1C per bit cell, except that the capacitor's dielectric is a multiferroic and its top electrode is a magneto-resistive stack. 
The incredible advantages promised by the implementation of multiferroics into RAM memories have led to the publication of other concepts of RAM utilizing multiferroic materials [109-111].

Bibes and Barthélémy suggested an improved design of MRAM, in which a multiferroic layer is utilized [110]. By choosing a multiferroic consisting of ferroelectric and antiferromagnetic phases, a hybrid device utilizing the exchange bias effect combined with the magneto-electric effect and the magneto-resistance could be employed. The key idea is to solve the main limitation of MRAM technology, which is related to the energy required in the "data write" process. Since large currents are necessary to create the switching magnetic fields, this naturally results in a substantial increase in the power consumption of the MRAM memory chip, as well as other issues associated with the Joule heating in the MRAM element.
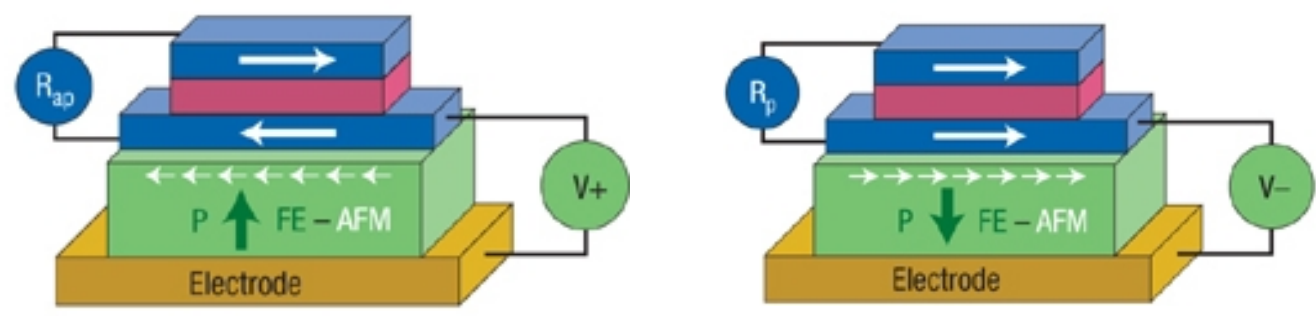

Figure 15. Image reprinted with permission from. Nature Materials 7, 425 - 426 (2008), article: Multiferroics: Towards a magnetoelectric memory, by Manuel Bibes, Agnès Barthélémy.

In their Nature paper, Bibes and Barthélémy proposed to use the interfacial exchange bias coupling between the anti-ferromagnetic phase of the multiferroic and a magnetic soft layer in order to induce the magnetization reversal and the two-resistance state of the MRAM element (figure 15).

This is achieved by controlling the exchange coupling at the interface through the application of a voltage, which has the effect to modify the spin structure at the interface due to the magneto-electric coupling effect. Essentially this design is a MRAM cell in which magnetization reversal (i.e. write operation) is controlled by an applied voltage rather than currents and magnetic fields.

This improves substantially the thermal stability of the device, the power consumption and the operation speed. However, unlike the previously proposed design, this is a non-volatile two-states RAM.

Gajek et al. reported the fabrication and study of magnetic tunnel junctions with multiferroic tunneling barriers [111]. They showed that multiferroic epitaxial $\mathrm{La}_{0.1} \mathrm{Bi}_{0.9} \mathrm{MnO}_{3}$ maintains ferroelectric and ferromagnetic states down to $2 \mathrm{~nm}$ thickness, which in turn allows its use as a quantum tunnel barrier in magnetic tunnel junctions. Their device has the

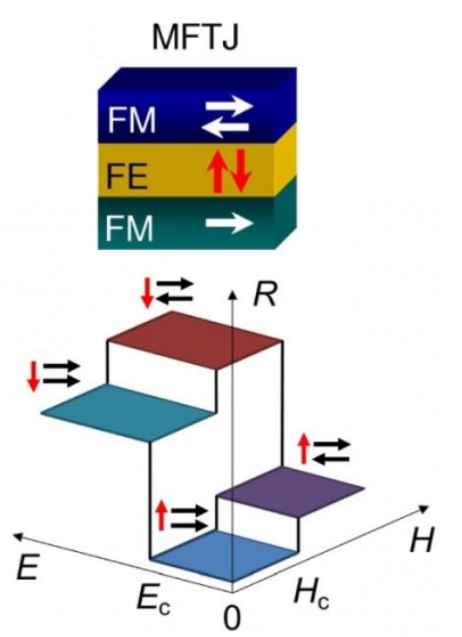

Figure 16. Schematic view of a multiferroic tunnel junction (MFTJ) with ferroelectric barrier. FM = ferromagnetic; $\mathrm{FE}=$ ferroelectric. Horizontal arrows indicate the direction of magnetizations and vertical arrows direction of electric polarization. Image reprinted with permission from MRS Buletin, Vol. 37,138 - 143 (2012) 
characteristics of a four-resistance-state memory system (figure 16). When the magnetizations of the magnetic electrode and multiferroic tunnel barrier are in parallel configuration a low resistance state is measured. When the two magnetizations are in antiparallel state the system is in high resistance state. However, two additional intermediary resistance states are also observed depending on the orientation of the electric polarization of the multiferroic tunnel barrier. Although their work represents an important step towards the ultimate memory element, it is worth mentioning that from practical point of view the fourstate-resistance memory requires cryogenic cooling for operation.

Gruverman et al. demonstrated using atomic force microscopy the tunnelling electroresistance effect on nano epitaxial $\mathrm{BaTiO}_{3}$ thin films deposited on $\mathrm{SrRuO}_{3}$. The obtained results show a substantial change in resistance upon polarization reversal at room temperature [112]. These results formed the foundation of possible future multiferroic tunnel junctions for non-volatile four-state memory RAM [113].

\subsection{Photovoltaic multiferroic solar cells}

The Sun radiates energy in all regions of the electromagnetic spectrum. Human eye is sensitive to a fraction of the solar spectrum ranging from 400 to $750 \mathrm{~nm}$, also called the visible spectrum. Although this is a very narrow region of the solar spectrum, it contains about $45 \%$ of all solar radiated energy, as can be seen in table 3 .

Table 3. Percentage of solar radiated energy per spectral region.

\begin{tabular}{|c|c|c|}
\hline Spectral region & Wavelength $(\mathbf{n m})$ & Percentage of solar radiated energy \\
\hline Infra red (IR) & $>\mathbf{7 5 0}$ & $\mathbf{4 6 . 3 \%}$ \\
\hline Visible (VIS) & $\mathbf{4 0 0 - 7 5 0}$ & $\mathbf{4 4 . 6 \%}$ \\
\hline Ultra Violet + (UV+) & $<\mathbf{4 0 0}$ & $\mathbf{9 . 1 \%}$ \\
\hline
\end{tabular}

Solar radiation is a fantastic source of energy that could be potentially harvested to power our planet. The solar power density at the Earth's surface is $1360 \mathrm{~W} / \mathrm{m}^{2}$, also known as the Solar Constant. Taking the Earth's radius $6350 \mathrm{Km}$, the total power received from the Sun is the solar constant times the area of a circle of radius equal to that of the Earth $\left(\pi \times 6.35^{2} \times 10^{12}\right.$ $\mathrm{m}^{2} \times 1360 \mathrm{~W} / \mathrm{m}^{2}=172,193 \times 10^{12} \mathrm{~W} \approx 173,000 \mathrm{TW}$ ). It is very instructive to compare this power with today's planetary power needs. Currently the worldwide rate of energy use is estimated to be around $16 \mathrm{TW}$ with the growth rate appearing to accelerate due to population growth and the rapid development of emerging economies. Excluding fossil fuel reserves, table 4 shows the global reserves in terms of the most important renewable energy sources.

Table 4. Global renewable energy reserves by source.

\begin{tabular}{ccccc}
\hline $\begin{array}{c}\text { Solar } \\
\text { radiation }\end{array}$ & Wind energy & Hydro & Geothermal & $\begin{array}{c}\text { Biomass } \\
\text { (50\% cultivable land) }\end{array}$ \\
\hline $173,000 \mathrm{TW}$ & $2-4 \mathrm{TW}$ & $2-4 \mathrm{TW}$ & $20-30 \mathrm{TW}$ & $7-10 \mathrm{TW}$ \\
\hline
\end{tabular}

Solar radiation is by far the most suitable candidate for renewable energy harvesting. Taking today's world power requirement, which is around $16 \mathrm{TW}$, and knowing that the solar power density at the surface of the Earth is $1360 \mathrm{~W} / \mathrm{m}^{2}$, then harvesting $100 \%$ of the solar energy, in 
order to power the entire planet, would require a surface of $\left.\mathrm{A}=\left(16 \times 10^{12}\right) / 1360\right) \mathrm{m}^{2}=$ $11,764 \mathrm{Km}^{2}$. Assuming a photovoltaic conversion efficiency of $10 \%$, then the entire planetary energy requirements would be fulfilled by a solar energy-harvesting power plant with a total surface of $117,640 \mathrm{Km}^{2}$. This is a surface equal to about half the surface area of the United Kingdom, which is around $243,000 \mathrm{Km}^{2}$, while the entire Sahara desert is about 4,619,260 $\mathrm{Km}^{2}$. With the Sun providing abundant, renewable clean energy, it is rather obvious that developing more efficient and cheaper photovoltaic technologies can solve the planetary energy needs regardless of the annual energy usage or population growth projections.

Photovoltaic effects typically involve two basic processes, including generation of electronhole pairs as the electrical-charge carriers, and separation of electrons and holes to form the net electric current flow in a particular direction.

Essentially all solar cells are made of standard p-n semiconductor junctions in which the electric current is produced by electron-hole pairs photo-excited across the band gap separating the conduction and the valence band [114]. The photovoltaic cells have usually an open circuit photo-voltage of $1 \mathrm{~V}$ or less, which is limited by the band gap and the energy barrier of the depletion layer at the p-n junction interface. Only photons with the energy larger than the band gap can produce electric current. Hence, engineering semiconductors with a small band gap will absorb more photons and produce larger current but small voltage. In contrast, semiconductors with a large band gap produce larger voltages but the current is limited because a large fraction of the solar photons are not absorbed. Therefore, existing semiconductor photovoltaic technology can harvest a small fraction of the solar spectrum, typically around $2 \mathrm{eV}$ visible photons. This paradigm is also known as the solar power dilemma [115].

One way of avoiding the dilemma of photovoltaic semiconductor solar cells hinges on a new technology, not based on p-n semiconductor junctions: the bulk photovoltaic effect [116] (see figure 17). This effect occurs in ferroelectric materials and its experimental evidence has already been reported in several perovskite ferroelectric oxide thin films such as PZT, $\mathrm{BaTiO}_{3}$ and $\mathrm{LiNbO}_{3}$ [117-119].

Although known since 1960, the physical mechanism of the bulk photovoltaic effect is not fully understood. A possible and widely accepted mechanism is given in figure 17. For a given poled ferroelectric crystal with internal polarization $P$, when photons with energies larger than the band gap of the dielectric ferroelectric material $\left(h v \geq E_{g}\right)$ are incident on the ferroelectric crystal, photons are absorbed resulting in the electrons and holes generation within the bulk of the ferroelectric crystal. Since the internal polarization $\mathrm{P}$ of the crystal has an associated internal electric field $\mathrm{E}\left(\mathrm{P} \approx \varepsilon_{0} \varepsilon_{\mathrm{r}} \mathrm{E}\right)$, the charge separation in the crystal takes place due to the internal E field driving the charge carriers towards the anode and cathode. Therefore, the photovoltaic effect in ferroelectrics is essentially a bulk effect, which is substantially different to the conventional interfacial effect in semiconductor $p-n$ junctions. In $\mathrm{p}-\mathrm{n}$ junctions, the charge separation occurs due to a field that exists only in a very narrow depletion region at the $\mathrm{p}-\mathrm{n}$ interface. The open circuit voltage in $\mathrm{p}-\mathrm{n}$ junctions cannot exceed the energy barrier of the junction itself, which is usually $\sim 1 \mathrm{~V}$.

In contrast, in the case of the bulk photovoltaic effect in ferroelectrics, the internal field $\mathrm{E}$ is a manifestation of the remanent polarization within the crystal and consequently exists over the whole bulk region of the ferroelectric crystal rather than within a thin interfacial region. Bulk photovoltaic effect has a charge transport that is not limited by the diffusion and an energy barrier does not limit the output photo-voltage. Studies of $\mathrm{LiNnO}_{3}$ ferroelectric crystals doped with $\mathrm{Fe}$ and $\mathrm{Cu}$ oxides revealed large photovoltaic effects when the samples were illuminated with $514 \mathrm{~nm}$ radiation. Photo-voltage as high as $1600 \mathrm{~V}$ has been measured in crystals 330 $\mu \mathrm{m}$ thick for light intensity of $150 \mathrm{~mW} / \mathrm{cm}^{2}$ [120]. In the same study, the photo-voltage 
dropped to $1000 \mathrm{~V}$ when the light intensity was reduced to $50 \mathrm{~mW} / \mathrm{cm}^{2}$. Similar results were later reported on studies of $\mathrm{BaTiO}_{3}$ ferroelectric crystals [121].

More recently, open circuit photo-voltage of $7 \mathrm{~V}$ was reported in PZT thin films doped with La [122]. Other studies on nano-structured PZT films doped with La reported unprecedented efficiencies of $0.28 \%$ in the bulk photovoltaic effect [123].

In spite of some clear advantages, the bulk photovoltaic effect in ferroelectrics has serious limitations such as:

i) low efficiency $\left(10^{-6}\right.$ to $\left.10^{-1}\right)$ due to the short lifetime of the photo-induced charge carriers;

ii) limited applicability due to the large band gap of oxide ferroelectric materials, which is typically $3-6 \mathrm{eV}$ making them efficient only in the high energy end of the solar spectrum (UV and beyond);

By replacing the ferroelectric material with a multiferroic that displays multiple order parameters (see figure 17), it is expected that the additional magnetic order parameter or the intrinsic strain coupling would lower the band gap of the structure making it photoactive in a wider solar spectrum. A bulk photovoltaic cell with a low band gap $(<1 \mathrm{eV})$ would ensure that the whole solar spectrum, or most of it, is utilized in the photovoltaic conversion process. Also, the $\mathrm{e}^{-}-\mathrm{e}^{-}$interactions governing the magnetic order can lead to smaller band gaps of typically $2.6-2.7 \mathrm{eV}$ for $\mathrm{BiFeO}_{3}$ multiferroic perovskites, as reported in [124-126].

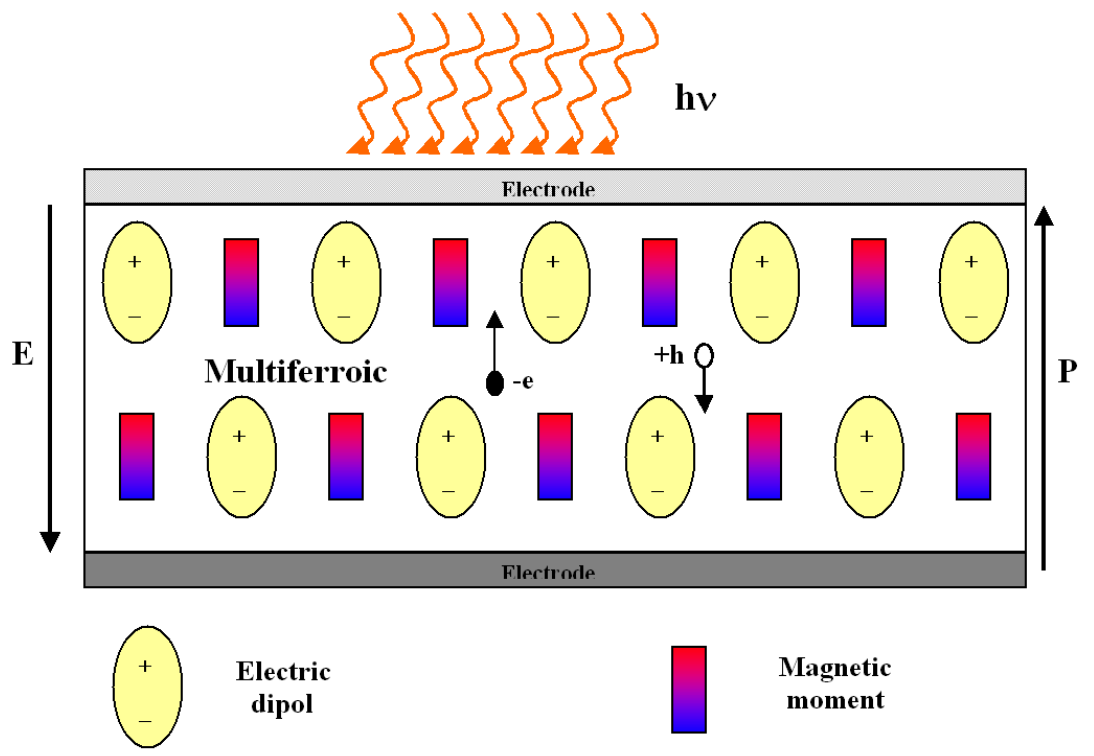

Figure 17. Schematic of bulk photovoltaic (PV) effect in multiferroics.

Moreover, recently published results reported large photovoltaic effects in $\mathrm{Cr}$ doped $\mathrm{BiFeO}_{3}$ multiferroics $\left(\mathrm{Bi}_{2} \mathrm{CrFeO}_{6}\right)$ thin films, with increased conversion efficiency when red laser illumination was used. The band gap of this multiferroic was measured to be around $1.4-2$ $\mathrm{eV}$. Although these theoretical and experimental studies are in their infancy, such low band gaps would result in bulk photovoltaic cells responsive to almost the whole solar spectrum. Measurements of the bulk photovoltaic effect in $\mathrm{Bi}_{2} \mathrm{CrFeO}_{6}$ multiferroic thin films reported in 2011 indicated an open circuit photo-voltage of $0.6 \mathrm{~V}$ under red light illumination with 1.5 $\mathrm{mW} / \mathrm{cm}^{2}$ intensity [127]. Moreover, the efficiency of this photovoltaic cell was estimated to be $\sim 6 \%$ when the thickness of the $\mathrm{Bi}_{2} \mathrm{CrFeO}_{6}$ multiferroic films was $125 \mathrm{~nm}$. 
It is not clear how the multiferroic state contributed to this massive improvement in both spectral response and efficiency, but it is believed that the $\mathrm{e}^{-}-\mathrm{e}^{-}$magnetic interactions can lower the band gap to useful levels.

In the same study, the photocurrent showed a clear response to electric polarization reversal (see figure 18), while the dark current was totally insensitive to polarization changes.

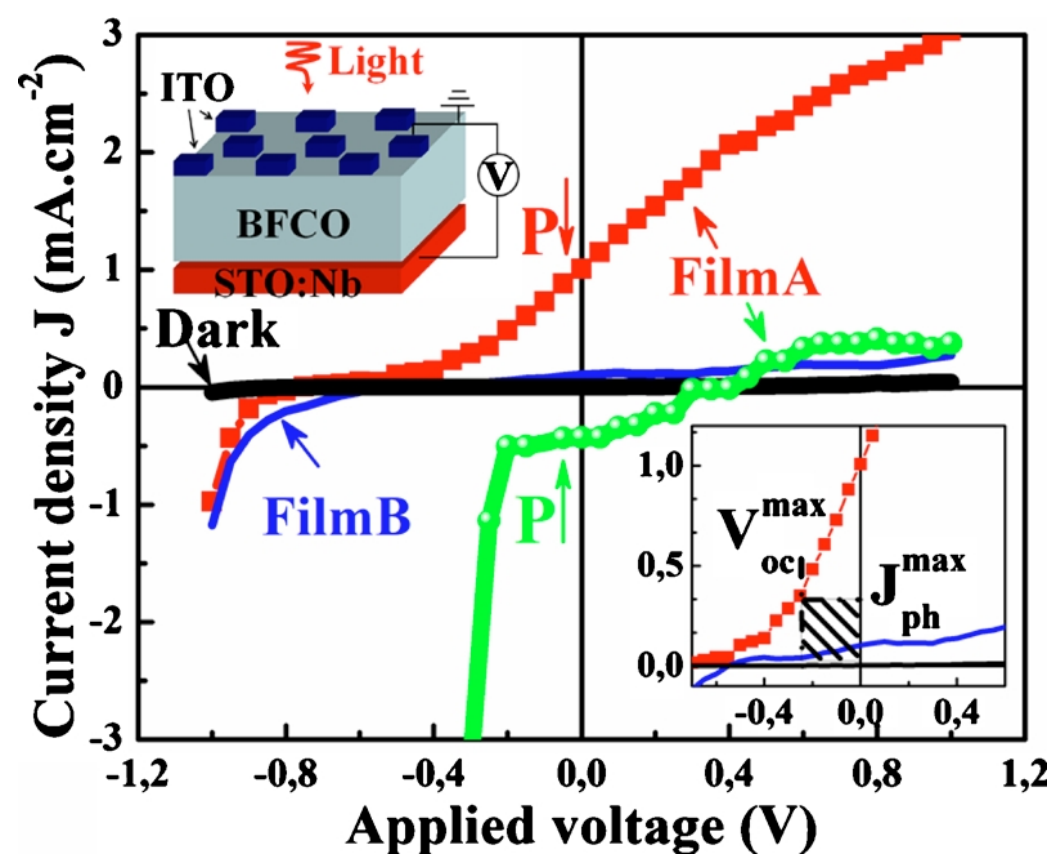

Figure 18. Dark and red light illumination $J-V$ measurements curves revealing a $\mathrm{PV}$ effect in the BFCO epitaxial films. The top-left inset shows a schematic of the device. The bottom-right inset is a zoom around zero indicating for film A the maximum current density and open circuit voltage used for the calculation of the efficiency. Image reprinted with permission from Appl. Phys. Lett. 98, 202902 (2011).

This is a further indication of the multiferroicity driven photo-response. In an ideal case, the multiferroic materials can be tuned to respond fully to the whole solar energy spectrum. This is possible because multiferroic materials can display interesting coupling effects between electric, magnetic and elastic order parameters. Hence, multiferroic material properties can be manipulated by externally applied magnetic fields, electric fields, stresses, as well as by the intrinsic strain coupling induced by the lattice mismatch between the multiferroic thin film and a substrate, as shown in this study [128]. This is an important open topic of research for both experimental and theoretical studies with far reaching social, economic and scientific consequences. Besides the obvious application to photovoltaic energy conversion, research of photovoltaic effects in multiferroics has revealed new effects such as the photostrictive effect [129], which opens up unlimited possibilities for applications based on opto-elasto-magnetoelectric active multiferroic materials.

\subsection{Multiferroics for thermal energy harvesting}

Heusler alloys, also known as shape memory alloys, are ferromagnetic inter-metallic materials that can change their shape when a magnetic field is applied. Hence, Heusler alloys are a special class of multiferroic materials, in which ferromagnetic and ferro-elastic phases 
coexist. In particular, these inter-metallic multiferroics are interesting as they undergo phase transformations in which diffusionless changes of the lattice parameters occur. These phase transitions lead to transformations from ferromagnetic austenite phase to non-ferromagnetic martensite phase. The large change in the lattice parameters that accompany these phase transitions are also associated with large entropy and internal energy changes. For this reason, such materials are very attractive for applications involving energy conversion, storage, shape memory actuation and solid-state refrigeration.

A good example is the NiTi shape memory alloy, which undergoes a reversible martensitic transformation and converts heat into mechanical work [130].

A)

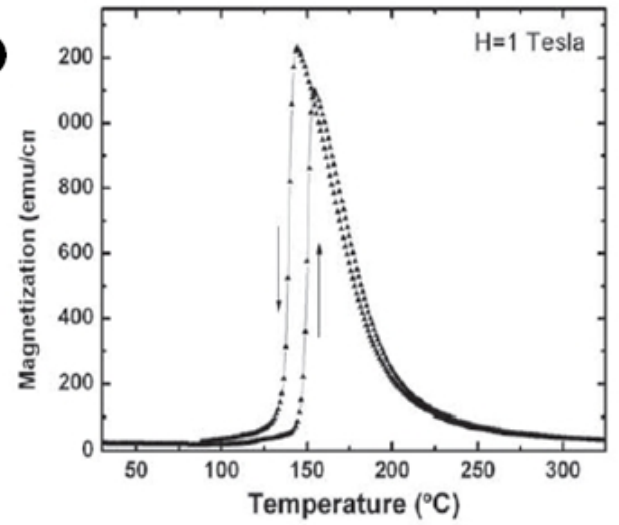

B)

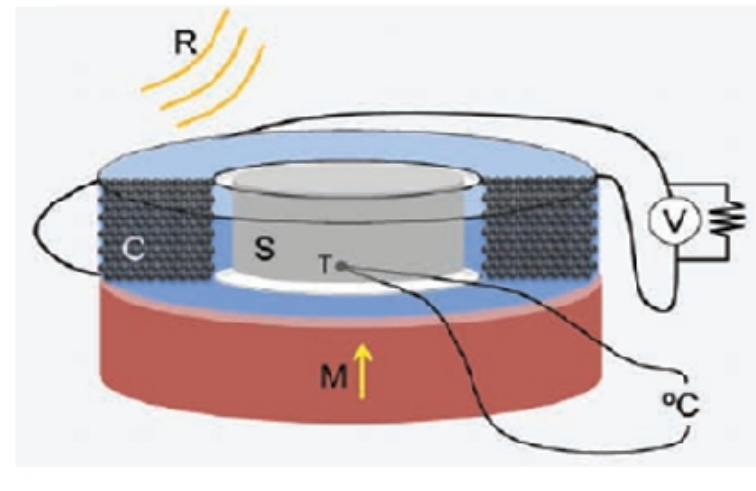

Figure 19. A) Magnetization vs temperature for $\mathrm{Ni}_{45} \mathrm{Co}_{5} \mathrm{Mn}_{40} \mathrm{Sn}_{10}$; B) Diagram of the thermal energy harvesting multiferroic device. $\mathrm{M}=$ magnetization; $\mathrm{C}=$ coil; $\mathrm{R}=$ heat source; $\mathrm{S}=$ specimen; $\mathrm{T}=$ thermocouple; V = voltmeter; Images reprinted with permission from Adv. Energy Mater. 1, 97-104 (2011).

Recently, the $\mathrm{Ni}_{45} \mathrm{Co}_{5} \mathrm{Mn}_{40} \mathrm{Sn}_{10}$ inter-metallic multiferroic alloy has been used for energy conversion, but unlike NiTi, it converts heat directly into electromotive work [131]. The $\mathrm{Ni}_{45} \mathrm{Co}_{5} \mathrm{Mn}_{40} \mathrm{Sn}_{10}$ inter-metallic multiferroic alloy displays low hysteresis, reversible martensitic phase transformation from non-magnetic to ferromagnetic phase upon heating [132].

If a suitable dc magnetic bias field biases the device, a sudden increase in the magnetic moment to a large value is observed during heating through the phase transition (figure 19A). The dc magnetic bias fields applied to the device were $0.5 \mathrm{~T}$ and $1 \mathrm{~T}$, respectively, although no substantial difference was observed between the two bias field values. At $1 \mathrm{~T}$ dc magnetic bias field, the phase transformation upon heating caused a remarkable increase of the magnetization from $10 \mathrm{emu} / \mathrm{cm}^{3}$ in the martensite phase to over $1100 \mathrm{emu} / \mathrm{cm}^{3}$ in the austenite phase. The sudden change in the magnetization is manifested as a flux changing magnetic field and it can be utilized to generate a voltage in a nearby coil via Faraday's induction. Hence, a direct conversion of heat into electricity mediated by the $\mathrm{Ni}_{45} \mathrm{Co}_{5} \mathrm{Mn}_{40} \mathrm{Sn}_{10}$ multiferroic alloy is achieved [131]. The thermal energy harvesting device developed by Srivastava et al. consists of a $\mathrm{Ni}_{45} \mathrm{Co}_{5} \mathrm{Mn}_{40} \mathrm{Sn}_{10}$ multiferroic specimen clamped near the pole of a permanent magnet and surrounded by 2000 turns $\mathrm{Cu}$ coil. A heat gun provided the heat flux and the potential induced was measured on a large $10 \mathrm{k} \Omega$ resistor placed between the ends of the $\mathrm{Cu}$ coil (figure 19-B). The output peak voltage of this energyharvesting device was $0.6 \mathrm{mV}$. 


\subsection{Multiferroic gyrators}

Investigation of electrical phenomena led to the creation of electronic elements, which are currently used to engineer advanced electronic and electrical applications. There are four well-known I-V linear electronic elements namely resistor, capacitor, inductor and the ideal transformer. In 1948, Bernard D. H. Tellegen, while working at the Philips Research Laboratories, proposed for the first time a hypothetical fifth linear element, known as the gyrator [133]. A gyrator is a linear, passive, lossless, two-port electrical element. However, its main feature is that, unlike the other four conventional linear elements, it violates the reciprocity theorem of ordinary networks. Hence, an important property of gyrators is that they invert the current-voltage characteristic of an electrical component or network, including the impedance in the case of linear elements. Figure 20-A shows the circuit symbol for the gyrator as originally invented by Tellegen [133]. An ideal two-port gyrator element is defined by the following equations:

$\mathrm{i}_{1}=\mathrm{G} \cdot \mathrm{v}_{2}$

$\mathrm{i}_{2}=-\mathrm{G} \cdot \mathrm{v}_{1}$

where $\mathrm{i}$ and $\mathrm{v}$ are the instantaneous current and voltage, respectively and the indexes represent the port number. The constant $G$ is called the gyration conductance and it has the dimensions of $1 / \mathrm{Ohm}$. It can be said that an ideal gyrator has the property that gyrates a current into a voltage and vice versa. For example, if we leave the secondary terminals open, $\mathrm{i}_{2}=0$, the primary terminals are short-circuited, $\mathrm{v}_{1}=0$ and vice versa. If an inductance $\mathrm{L}$ is connected to the secondary terminals, between the primary terminals a capacitance is formed, $\mathrm{C}=\mathrm{L} \cdot \mathrm{G}^{2}$. If a capacitance $\mathrm{C}$ is connected to the secondary terminals, between the primary terminals we find an inductance, $\mathrm{L}=\mathrm{C} / \mathrm{G}^{2}$. Finally, if a resistor of impedance $\mathrm{Z}$ is placed in the secondary, then the primary input port behaves as a linear resistor with resistance $1 / \mathrm{G}^{2} \cdot \mathrm{Z}$. Because a gyrator can make an inductive circuit behave capacitively and vice versa, its primary application is to reduce the size and cost of a system by removing the need for bulky, heavy and expensive inductors. Gyrator circuits are extensively used in active filter design, miniaturization and telephony devices.

Today's circuits that function as gyrators can be built with transistors and operational amplifiers using feedback. However, Tellegen suggested a number of ways in which a practical gyrator might be built. In fact, it can be argued that Tellegen is the original inventor not only of the gyrator, but also of the multiferroic materials and their magneto-electric effect. In his quest to identify a gyrator medium, Tellegen has independently invented the multiferroic materials and the linear magneto-electric effect in 1948. Indeed, in section 8 of his 1948 article, Tellegen wrote that a gyrator could be constructed if a medium that fulfills the following relations: $\mathrm{D}=\varepsilon \mathrm{E}+\gamma \mathrm{H}$ and $\mathrm{B}=\gamma \mathrm{E}+\mu \mathrm{H}$, is identified. While $\mathrm{D}=\varepsilon \mathrm{E}$ and $\mathrm{B}=$ $\mu \mathrm{H}$ are well understood ( $\varepsilon$ is the dielectric permitivity and $\mu$ is the magnetic permeability), Tellegen himself wrote, "the coefficient $\gamma$ is something new" (see page 402 in ref. [133]).

Of course, today it is well known that $\gamma$ is in fact the linear magneto-electric coupling coefficient of multiferroics, for which we use the $\alpha$ symbol. Tellegen also gave a relationship between the magneto-electric coupling and the magnetic and electric susceptibilities, $\gamma^{2} / \varepsilon \mu \leq$ $\gamma^{2} / \chi^{\mathrm{e}} \chi^{\mathrm{m}} \leq 1[133]$ 
A)

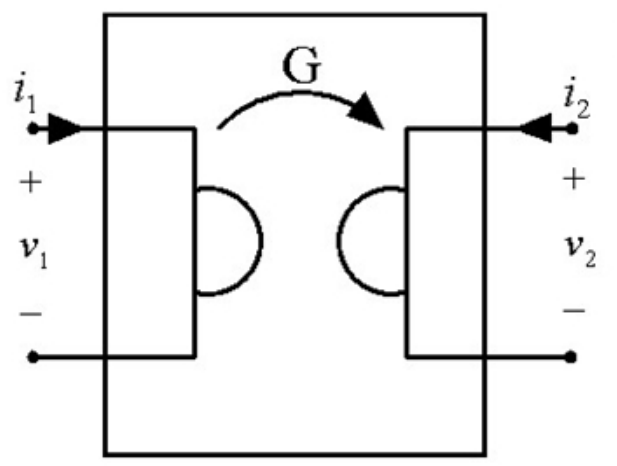

B)

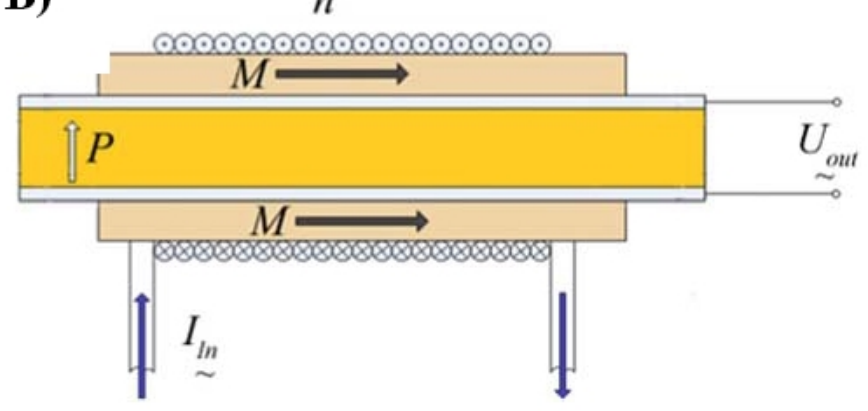

Figure 20. A) Circuit symbol of the gyrator, as proposed by Tellegen in Philips Res. Rept. 3, 81-101 (1948)]; B) Schematic diagram of the multiferrouic gyrator proposed by Zhai et al. Image reprinted with permission from Eur. Phys. J. B 71, 383-385 (2009)

Figure 20-B shows the schematic diagram of the multiferroic gyrator proposed by Zhai et al. [134]. The authors fabricated three different tri-layer laminated composite multiferroics : i) a Terfenol-D/PZT/Terfenol-D structure with electromechanical resonance frequency $\mathrm{f} \approx 86$ $\mathrm{kHz}$; ii) a Metglas/PZT/Metglas structure with electromechanical resonance frequency $\mathrm{f} \approx 64$ $\mathrm{kHz}$; iii) and a Nickel/PZT/Nickel composite multiferroic with electromechanical resonance frequency $\mathrm{f} \approx 15 \mathrm{kHz}$; In order to function as a gyrator, the tri-layer composite multiferroic structures were wrapped tightly in a coil circled around the laminated structure. An applied current to the coil generates the ac magnetic field acting on the magneto-strictive magnetic layers. The strain mediated magneto-electric coupling is therefore induced and a voltage output is collected from the piezo-layer. The input current to the coil corresponds to port 1 of the gyrator and the output voltage from the piezo-component corresponds to port 2 of the gyrator (see figure 20). The experimental demonstration of the multiferroic gyrator opens up the possibility of further miniaturization of electrical networks and circuits by replacing current multi-component electronic gyrator circuits with a single multiferroic element.

\subsection{Multiferroics for solid state cooling: the multicaloric effect}

Modern refrigeration is based almost entirely on a compression / expansion cooling cycle and has the following limitations: i) low Carnot efficiency of $<60 \%$; ii) utilizes refrigerants that are usually hazardous chemicals, ozone depleting and greenhouse gases; iii) very difficult to miniaturize for integration into silicon chips; iv) unsuitable for ultra-low (mK - $\mu \mathrm{K})$ cryogenic cooling;

Unsurprisingly, these disadvantages are driving the market away from this technology and solid-state cooling is a strong competitor because it is a clean and energy efficient technology that utilizes the adiabatic relaxation of an order parameter in solids (electric, magnetic or elastic) to produce a temperature reduction of the solid refrigerant.

Most common solid-state cooling technology today is based on the magneto-caloric effect, which was first proposed theoretically by Debye (1926) and Giauque (1927), and demonstrated experimentally seven years later [135]. Today, magneto-caloric cooling is available commercially mainly for space cooling [136]. Temperatures as low as $10-40 \mathrm{mK}$ are easily achievable using Cerium Magnesium Nitrate $\left(\mathrm{Ce}_{2} \mathrm{Mg}_{3}\left(\mathrm{NO}_{3}\right)_{12} \cdot 24 \mathrm{H}_{2} \mathrm{O}\right.$ paramagnetic salts, and $22 \mu \mathrm{K}$ have been reported in $\mathrm{PrNi}_{5}$ paramagnetic intermetallic alloys [137]. 
Magneto-caloric refrigeration at room temperature is almost entirely based on Gadolinium alloys [138] with $6-13 \mathrm{~K}$ adiabatic temperature changes reported. However, such performance requires magnetization - demagnetization cycles of very large applied magnetic fields $(50-100 \mathrm{kOe})$. This makes magneto-caloric cooling inaccessible to the mass consumer market, unless new permanent magnets generating very large magnetic fields are developed, or new caloric materials and effects are discovered. In this respect, a few other caloric effects and materials have been discovered, most notable: electro-caloric [139], elasto-caloric [140] and toroido-caloric effect [141], all showing some promising results, but still not competitive enough to replace magneto-caloric or compression/expansion refrigeration.

Recent developments in solid-state caloric effects led to the realization that traditional solidstate cooling via electro-caloric, magneto-caloric or elasto-caloric materials could be further improved when cross-coupling effects between the ferroic phases within these materials stimulate large entropy changes. Experimental evidence of the cross coupling effects have been published by Moya et al. showing that a $\mathrm{La}_{0.7} \mathrm{Ca}_{0.3} \mathrm{MnO}_{3}$ perovskite thin films show poor magneto-caloric effects and giant magneto-caloric properties when strain coupled magneto-elastically to a suitable substrate [142]. Other ideas of caloric effects enhanced via the magneto-electric coupling in multiferroic materials were also proposed recently. Vopson proposed in fact a new promising caloric effect that occurs only in multiferroic materials, called the multicaloric effect [143].

Although the theory of the multicaloric effect was first published in 2012 [143], the term multicaloric was coined by Fahler et al. in an earlier article [144]. However, while Fahler et al. recognized the possibility of an enhanced multicaloric effect via cross-couplings in multiferroics, the authors made in their article no attempt to develop the theory or to report any experimental work on this topic. After the publication of the multicaloric effect, other articles followed shortly, reporting similar findings $[145,146]$. This culminated with the publication in 2013 of the theoretical generalization of the multicaloric effect [147]. In this article, a general formula has been proposed, from which all possible caloric effects including cross-coupling effects can be derived.

Essentially, the multicaloric effect predicts that multiferroic materials can display giant caloric effects significantly greater to those achieved in magneto-caloric, electro-caloric or elasto-caloric materials alone. The relations describing the multicaloric effect induced electrically and magnetically in magneto-electric multiferroics are:

$$
\begin{aligned}
& \Delta T_{e}=-\frac{T}{C} \cdot \int_{E_{i}}^{E_{f}}\left[\frac{\alpha_{e}}{\mu_{0} \chi^{m}} \cdot\left(\frac{\partial M}{\partial T}\right)_{H, E}+\left(\frac{\partial P}{\partial T}\right)_{H, E}\right] \cdot d E \\
& \Delta T_{m}=-\frac{T}{C} \cdot \int_{H_{i}}^{H_{f}}\left[\left(\frac{\partial M}{\partial T}\right)_{H, E}+\frac{\alpha_{m}}{\varepsilon_{0} \chi^{e}} \cdot\left(\frac{\partial P}{\partial T}\right)_{H, E}\right] \cdot d H
\end{aligned}
$$

where $\mathrm{T}$ is the temperature, $\mathrm{C}$ is specific heat capacity of the system per unit volume $C=$ $T \cdot(\partial S / \partial T)_{H, E}, \mu_{0}, \varepsilon_{0}$ are the magnetic permeability and dielectric permittivity of vacuum, $\chi^{m}$ and $\chi^{e}$ are the magnetic and electric susceptibilities, $\alpha_{e}$ and $\alpha_{m}$ are the electrically and magnetically induced magneto-electric coupling coefficients, $\mathrm{M}$ is magnetization and $\mathrm{P}$ is electric polarization of the multiferroic system. A full derivation of the multicaloric effect is given in $[143,147]$.

Relations (25), (26) show that a finite adiabatic change in the applied external fields $E$ or $H$ produce a temperature change $\Delta T$, which for a multiferroic system is larger than the equivalent temperature change corresponding to an electro-caloric or magneto-caloric material subjected to the same applied $E$ and $H$ fields, respectively. According to (25), (26) it 
can be easily shown that a heating effect $(\Delta T>0)$ is achieved for an adiabatic polarization / magnetization and a cooling effect $(\Delta T<0)$ for an adiabatic depolarization / demagnetization, respectively.

The proposed operation principle of a solid-state cooling cycle based on the multicaloric effect in a magneto-electric multiferroic is shown diagrammatically in figure 21 , together with the associated Brayton thermodynamic cooling cycle. This is based on the fact that electric dipoles and magnetic spins / moments in multiferroic solids can absorb heat from the environment via a relaxation process as following:

a) The multicaloric cooling cycle begins with the system initially in thermal equilibrium at temperature $T$, where magnetic spins / moments and electric dipole moments fluctuate randomly in a para-multiferroic state due to the thermal activation;

b) Under adiabatic conditions, a strong $H$ or $E$ field is applied to the refrigerant multiferroic system, forcing the magnetic spins and electric dipole moments to align, causing a transition from disorder to ordered multiferroic state (A-B on the Brayton cooling cycle). This, decreases the entropy of the system, which under adiabatic conditions will cause the system to heat up to $T+\Delta T_{a d}$;

c) The added temperature can then be removed via a heat sink, while the applied field is kept constant to prevent the spins and electric dipoles reabsorbing heat. The system then returns to the equilibrium temperature $T$ given by the heat sink (transition B-C on the Brayton cycle);

d) Thermal contact with the heat sink is then broken via a thermal switch so the system is adiabatic, while simultaneously the applied $H$ or $E$ field is switched off (transition $\mathrm{C}-\mathrm{D}$ in the Brayton cycle). This is an adiabatic demagnetization and depolarization process, which causes the spins and electric dipoles to absorb the heat in their relaxation process, decreasing the temperature of the refrigerant below the temperature of the heat sink, $T-\Delta T_{a d}$;

e) The multiferroic refrigerant can then be placed in thermal contact with the environment being refrigerated (transition D-A on the Brayton cycle) and the whole cycle is repeated again.

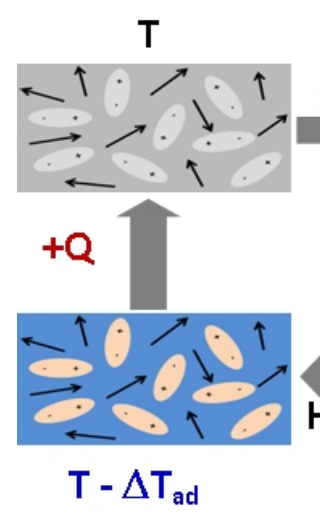

A)

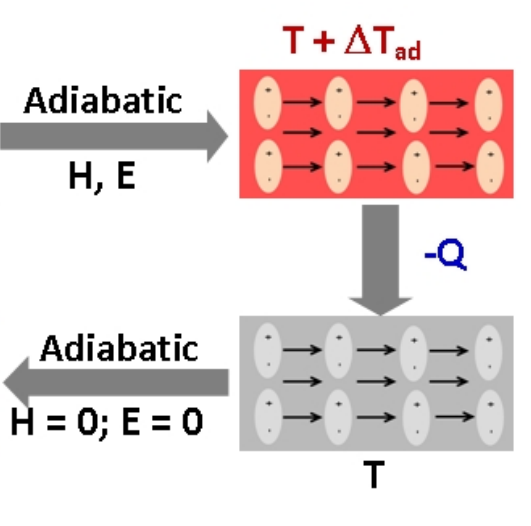

B)

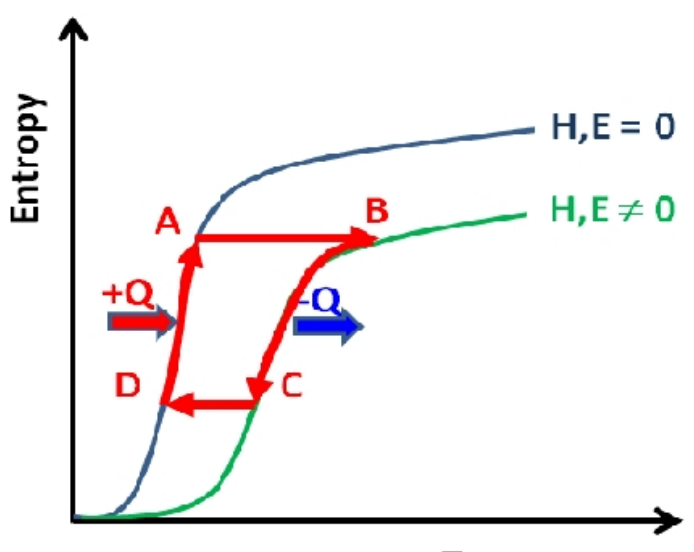

Temperature

Figure 21. Diagram of the refrigeration cycle proposed in the case of solid-state multiferroic cooling via the multicaloric effect. A) Schematic of the four cooling stages (A-D) showing the entropy change due to relaxation of the magnetic moments (black arrows) and electric dipoles (ovals); B) The corresponding Brayton cooling cycle. Image reprinted with permission from Journal of Physics D: Applied Physics, 46 (34) (2013) 
Initial numerical estimations of the multicaloric effect in $\mathrm{NdCrTiO}_{5}$ polycrystalline multiferroic material have been performed using data extracted Hwang et al. paper [45]. Calculations indicated an adiabatic temperature change of $\sim 7 \mathrm{~K}$ per cycle [143], calculated for magnetically induced multicaloric effect at the operation temperature of $21 \mathrm{~K}$ and the adiabatic magnetization / demagnetization under 5 to 0 Tesla magnetic field cycles. Around $10 \%$ of the temperature change was due to the magneto-electric coupling term specific to the multicaloric effect. Although Midya et al. already reported giant caloric effects in $\mathrm{R}-\mathrm{MnO}_{3}$ multiferroics [148], the multicaloric effect is yet to be confirmed experimentally.

\subsection{Other possible applications of multiferroics}

It is important to note that a few of the applications covered in detail here, are also briefly mentioned in the 1974 review article published by Wood and Austin [18]. In the same paper, the authors suggested other applications that are not discussed in this review, mostly in the area of optical and electro-optical devices (both linear and non-linear) operating in visible, infra-red and microwave spectral region (see Table 1 in ref. [18]).

Although in this article the main focus is on the linear coupling effects in multiferroics and their possible applications, it is important to mention that the occurrence of a non-linear magneto-electric effect has been experimentally observed and utilized in novel frequency multiplier and frequency mixer devices based on multiferroic composite laminates [149, 150]. Besides the applications of multiferroic materials already presented in detail in sections 5.1 to 5.10 , there are other proposed applications that deserve brief mentioning in this section and, without any doubt, many more will emerge in future.

\subsubsection{High voltage gain multiferroic amplifier}

Dong et al. realized a high voltage gain multiferroic amplifier [151]. This is essentially a trilayer multiferroic composite with the structure Terfenol-D / PZT / Terfenol-D shaped in an elongated bar geometry. The novelty is that the PZT component is in fact made up of two elements longitudinally poled and placed in reverse directions to each other to maximize the voltage output of the device. A solenoid coil is wrapped around the tri-layer multiferroic bar structure, in a similar way to the gyrator structure. A voltage input to the coil will result in the generation of ac magnetic fields to the device, which in turn initiate the strain mediated magnetically induce magneto-electric effect and a voltage output is collected from the PZT component. Dong et al. demonstrated a voltage gain $\mathrm{V}_{\text {out }} / \mathrm{V}_{\text {in }} \approx 300$, when the device operates at the resonance frequency, $f=21.3 \mathrm{kHz}$, and under a dc magnetic bias field of 300 Oe [151].

\subsubsection{Multiferroic magnetic gradiometer}

Bedekar et al. designed a magnetic gradiometer based on a bi-layer multiferroic composite consisting of a ring-dot piezoelectric transformer structure and a magnetostrictive layer bonded on top of the ring-dot structure. When the device is operated near the electromechanical resonance frequency, the authors demonstrated the gradiometer functionality [152], in which the magnetic field gradient is measured through the frequency shift and output voltage change of the device. Again, terfenol-D and PZT were used and the device had a working resonance frequency of $91-94 \mathrm{kHz}[152]$.

\subsubsection{Vortex magnetic field detector based on multiferroics}

Instead of planar layered sensor, by constructing the multiferroic composite laminate into a ring shape, a vortex magnetic field detector or current sensor can be achieved $[76,153]$. 
Indeed, a current passing through a straight wire will generate a vortex magnetic field around the wire. The field generated is linear with the current, so a ring-shape multiferroic detector would act as a vortex field and current detector. Using a PZT ring consisting of multiple segments poled in the circumference direction and sandwiched in between two terfenol-D rings magnetized in the circumference, Dong et al. realized a highly sensitive magnetic vortex field and current sensor which had a response of up to 100 times higher than 100 turns reluctance coil used for the same measurements [76].

\subsubsection{Vibration energy harvesting multiferroics}

Another technology sector where multiferroics offer promising results is the energy harvesting of ambient vibrations [154]. Devices capable to harvest energy from environmental sources are of huge commercial interest due to their suitability to power wireless transducer and sensor networks, environmental condition monitoring systems, structural health monitoring systems, security systems, medical implants and portable electronics. The main driving force of energy harvesting industry is the fact that in many applications the limited lifespan of batteries increases the maintenance costs, especially in harsh environment locations where access is restricted or hazardous. This is a matter of vital relevance in the case of medical implants powered by batteries that require secondary surgeries for replacement, increasing substantially the costs and the patient health risks. On the other hand, micro-energy harvesting devices are low cost, long lifespan and require little or no maintenance. The main technology for vibration energy harvesting is currently based on piezoelectric devices [155-158], a growing industry predicted to reach 145 million UDS in 2018 from around 20 million USD market in 2013 [www.energyharvestingjournal.com].

Besides the piezoelectric effect, other ways of harvesting mechanical vibrations based on the magnetostrictive vibration energy harvesting have been proposed [159]. In this case, mechanical motion induces sharp magnetization changes in a strongly magnetostrictive material. An inductive coil, via the Faraday effect, then picks up such magnetization changes converting the mechanical motion directly into an electromotive force [160].

Combining the piezo-energy harvesting and magnetostrictive energy harvesting of mechanical vibrations within a single multiferroic device could result in an ultra-efficient multiferroic vibration energy harvester. This idea has already been implemented and promising results of hybrid piezoelectric / magnetostrictive multiferroic energy harvesters have been already reported [161].

\subsubsection{Magnetic data storage based on multiferroics}

Kim et al. demonstrated experimentally a novel concept of controlling super-paramagnetism via the magneto-electric effect in multiferroics [162]. In their study, the multiferroic composite system consisted of $\mathrm{Ni}$ magnetic nanoparticles strain coupled to a piezo-electric $\left[\mathrm{Pb}\left(\mathrm{Mg}_{1 / 3} \mathrm{Nb}_{2 / 3}\right) \mathrm{O}_{3}\right]_{(1-\mathrm{x})}-\left[\mathrm{PbTiO}_{3}\right]_{\mathrm{x}}(\mathrm{PMN}-\mathrm{PT}, \mathrm{x}=0.3)$ substrate. Unpoled substrate left the $\mathrm{Ni}$ nano-particles in super-paramagnetic state, while the application of an electric field switched the Ni particles to magnetized state. These results were adopted in a follow up study by Vopson et al., in which they proposed to use this effect in a multiferroic composite magnetic data storage medium for areal densities beyond the super paramagnetic limit [163]. Through modeling of thermal stability of these systems, the authors demonstrated how such multiferroic data storage medium could lead to unprecedented increases in data storage areal density beyond the super-paramagnetic size limit and potentially replace Heat Assisted Magnetic Recording (HAMR) technology [163]. 


\section{Future directions and concluding remarks}

In conclusion, multiferroic materials are a huge source of technological applications because they can simultaneously display tunable mechanical, magnetic, electric/dielectric, thermal and optical properties. These parameters can also couple to each other giving rise to interesting cross-coupling effects that further enhance the versatility of multiferroics for novel applications. Depending on the area of applicability, different multiferroic material requirements apply to different applications. In most cases however, common requirements that must be strictly observed for applications are: a) multiferroic materials must display large magneto-electric coupling effects, preferably at room temperature; b) multiferroic materials and devices must be easy to manufacture and preferably consisting of cheap chemical elements and compounds; c) multiferroic structures must be suitable for large scale production and integration at chip / wafer level; d) multiferroic structures must be suitable for fabrication at micro- and nano-scale sizes, without loss of functionality. In fact, there is already experimental evidence that multiferroic magneto-electric coupling is preserved even at nano-scale [164], suggesting that nano-technologies based on these materials are indeed possible.

Most of the requirements listed above are fulfilled today by composite multiferroic materials. Indeed, the research field of composite multiferroics continues its steady growth with new ideas and structures being frequently published, including the recent emergence of flexible PVDF-polymer based multiferroic composites [165] and their applications [166]. Despite major advances in composite multiferroics, a much-awaited scientific breakthrough is the discovery and synthesis of a single-phase multiferroic compound optically active, piezoactive, displaying magnetic and electric order phases and large cross-coupling coefficients at room temperature. Such compound would be the basis of the so-called "all-in-one universal solid-state element", capable of mechanical actuation, multiple memory states, logic functions, sensing and photoactive properties within a single multiferroic multifunctional solid-state entity with unprecedented versatility for high-tech applications. With concentrated research efforts, the discovery of such material is very plausible.

It is not an overstatement to suggest that multiferroic materials have the potential to fundamentally transform our society and to allow the current scientific and technological progress to continue. If only a fraction of the proposed applications will materialize in commercial products, multiferroic technologies could soon reach multibillion US dollar market value. Moreover, the merger of multiferroics with semiconductors and spintronic materials forming multifunctional hybrid structures could facilitate the emergence of truly disruptive new technologies.

\section{Acknowledgements}

The author would like to acknowledge the direct and indirect support to this work received from various entities including the University of Portsmouth, the UK National Physical Laboratory and Seagate Technology - Northern Ireland. In particular, the author would like to express special thanks to the Faculty of Science, University of Portsmouth for facilitating the introduction of his new third year Physics Unit entitled "Introduction to multiferroic materials and their applications", which is the basis of this review article. 


\section{References}

[1] D.N. Astrov, J. Exp. Theoret. Phys. (U.S.S.R) 38, 948 (1960)

[2] V.J. Folen, G.T. Rado, E.W. Stader, Phys. Rev. Lett. 6 (1961) 607

[3] G.T. Rado, V.J. Folen, Phys. Rev. Lett. 7 (1961) 310

[4] A.K. Agyei, J.L. Birman, J. Phys: Condens. Matter 2, 3007-3020 (1990)

[5] M. Fiebig, J. Phys. D: Appl. Phys. 38 (2005) R123

[6] G. Lawes, G. Srinivasan, J. Phys. D.: Appl. Phys. 44, 243001 (2011)

[7] J.P. Rivera, Ferroelectrics, vol 161 (1994) 165-180

[8] G. Srinivasan, Annual Review of Materials Research 40, 153-178 (2010)

[9] W. Prellier, M.P. Singh, P. Murugavel, J. Phys: Condens. Matter 17, R803-R832 (2005)

[10] C.W. Nan, M.I. Bichurin, S. Dong, D. Viehland, G. Srinivasan, J. Appl. Phys. 103 (3), 031101 (2008)

[11] M. Vopsaroiu, M. Stewart, T. Hegarty, A. Muniz-Piniella, N. McCartney, M. Cain, G. Srinivasan, Measurement Science \& Technology, 19, 4 (2008)

[12] Hans Schmid, Ferroelectrics 162, 317-338 (1994)

[13] P. Curie, J. Physique, 3, 393 (1894)

[14] P. Debye, Z. Phys. 36, 300 (1926)

[15] I.E. Dzyaloshinskii, Sov. Phys. - JETP 10, 628 (1959)

[16] T.H. O’Dell, Phil. Mag. Vol. 7, Issue 82, (1962)

[17] T.H. O'Dell, The electrodynamics of magneto-electric media, Amsterdam: North - Holland (1970)

[18] V.E. Wood, A.E. Austin, Int. J. Magn. 5, 303 (1973)

[19] J. F. Scott "Applications of Magnetoelectrics" J. of Materials Chemistry, 2012, v. 22, paper 4567

[20] A.V. Shubnikov, Symmetry and antisymmetry of finite figures, Moscow: USSR Acad. Sci. (1951)

[21] L.V. Shuvalov, N.V. Belov, Kristallographya 7, 192 (1962)

[22] L.V. Shuvalov, N.V. Belov, Sov. Phys. Crystallogr. 7, 150 (1962)

[23] R.R. Birss, Symmetry and magnetism, Amsterdam: North-Holland, (1966)

[24] H. Schimd, J. Phys. C: Condens. Matter 20, 434201 (2008)

[25] Nicola A. Hill, J. Phys. Chem. B, 104, $6694-6709$ (2000)

[26] D. Khomskii, Physics 2, 20 (2009)

[27]C.W. Nan, L.Liu, N. Cai, J. Zhai, Y. Ye, Y.H. Lin, L.J. Dong, C.X. Xiong, Appl. Phys. Lett. 81 (2002) 3831

[28] A.M. van Run, D.R. Terrell, J.H. Scholing, J. Mater. Sci. 9, 1710-1714 (1974)

[29] N. Cai, C.W. Nan, J. Zhai, Y. Lin, Appl. Phys. Lett. 84 (2004) 3516

[30] J. Ryu, A.V. Carazo, K. Uchino, H.E. Kim, Jpn. J. Appl. Phys. Part 1, 40, 4948 (2001)

[31] G. Srinivasan, E. T. Rasmussen, B. J. Levin, and R. Hayes, Phys. Rev. B 65 (2002) 134402

[32] M.K. Lee, T.K. Nath, C.B. Eom, M.C. Smoak, F. Tsui, Appl. Phys. Lett. 77 (2000) 3547

[33] S.S. Kim, J.W. Lee, S.C. Shin, H.W. Song, C.H. Lee, K. No, J. Magn. Magn. Mater. 267 (2003) 127

[34] G. Srinivasan, E. T. Rasmussen, J. Gallegos, R. Srinivasan, Yu. I. Bokhan, and V. M. Laletin, Phys. Rev. B 64 (2001) 214408

[35] U. Laletsin, N. Padubnaya, G. Srinivasan, C.P. Devreugd, Appl. Phys. A 78 (2004) 33

[36] G. Srinivasan, E. T. Rasmussen, R. Hayes, Phys. Rev. B 67 (2003) 014418

[37] M. Vopsaroiu, M.G. Cain, G. Sreenivasulu, G. Srinivasan, A.M. Balbashov, Materials Letters 66, 282-284 (2012)

[38] J. Ma, Y. Lin, C.W. Nan, J. Phys. D: Appl. Phys. 43, 012001 (2010)

[39] D.A. Pan, S.G. Zhang, A.A. Volinsky, L.J. Qiao, J. Phys. D: Appl. Phys. 41, 172003 (2008)

[40] R. Jahns, A. Piorra, E. Lage, C. Kirchhof, D. Mayners, J.L. Gugat, M. Krantz, M. Gerken, R. Knochel, E. Quandt, J. Am. Ceram. Soc., Vol. 96, Issue 6, 1673-1681 (2013)

[41] T.R. McGuire, E.J. Scott, F.H. Grannis, Phys. Rev. 102 (1956) 1000

[42] J.P. Rivera, Ferroelectrics, vol 161 (1994) 165-180

[43] G.T. Rado, Phys. Rev. Lett. 13 (1964) 335

[44] J.P. Rivera, Ferroelectrics, vol 161 (1993) 147

[45] J. Hwang, E.S. Choi, H.D. Zhou, J. lu, P. Schlottmann, Phys. Rev. B, 85, 024415 (2012)

[46] G.T. Rado, J.M. Ferrari, W.G. Maisch, Phys. Rev. B 294041

[47] B.B. Krichevtsov, V.V. Pavlov, R.V. Pisarev, JETP Lett. 49 (1989) 535

[48] J. Wang, J.B. Neaton, H. Zheng, V. Nagarajan, S.B. Ogale, B. Liu, D. Viehland, V. Vaithyanathan, D.G. Schlom, U.V. Waghmare, N.A. Spaldin, K.M. Rabe, M. Wuttig, R. Ramesh, Science vol 299 (2003) 1719

[49] J. Hemberger, Nature 434 (2005) 364

[50] M.E. Lines, A.M. Glass, Principles and Applications of Ferroelectrics and Related Materials, 1977

[51] H. Sugie, N. Iwata, K. Kohn, J. Phys. Soc. Japan, 71 (2002) 1558

[52] T. Kimura, T. Goto, H. Shintani, K. Ishizaka, T. Arima, Y. Tokura, Nature 426 (2003) 55 
[53] N. Hur, S. Park, P.A. Sharma, J.S. Ahn, S. Guha, S-W. Cheong, Nature 429 (2004) 392

[54] D. Fox, J.F. Scott, J. Phys. C 10 (1977) L329

[55] T. Kimura, G. Lawes, G.A.P Ramirez, Phys. Rev. Lett. 94 (2005) 137201

[56] V.R. Palkar, S.K. Malik, Solid State Com. 786134 (2005) 783-786

[57] Y. Wang, J. Hu, Y. Lu, Ce-Wen Nan, NPG Asia Materials 2 (2) 61-68 (2010)

[58] J. Zhai, Z. Xing, S. Dong, J. Li, D. Viehland, J. Am. Ceram. Soc. 91 (2) 351-358 (2008)

[59] R.E. Newnham, D.P. Skinner, L.E. Cross, Mater. Res. Bull. 13, 525 91978)

[60] R. Ramesh, N. A. Spaldin, Nature Materials, vol. 6, 21-29 (2007)

[61] M. Vopsaroiu, M.J. Thwaites, S. Rand, P.J. Grundy, K. O’Grady, IEEE Trans. Magn. 40, 2443 (2004)

[62] M. Vopsaroiu, G.V Fernandez, M.J. Thwaites, J. Anguita, P.J. Grundy, K. O'Grady, J. Phys. D: Appl. Phys. vol. 38, pp. 490-496, (2005)

[63] J. van Suchtelen, Phylips Res. Rep. 27, 28 (1972)

[64] J. Boomgard, D.R. Terrell, R.A.J. Born, H.F.J.I. Giller, J. Mater. Sci. 9, 1705 (1974)

[65] J. Boomgard, A.M.J.G. Run, J. Suchtelen, Ferroelectrics, 10, 295 (1976)

[66] J. Boomgard, R.A.J. Born, J. Mater. Sci. 13, 1538 (1978)

[67] G. Harshe, J.P. Dougherty, R.E Newnham, Int. J. Appl. Electromagn. Mater. 4, 145 (1993)

[68] S. lopatin, I. Lopatin, I. Lisnevskaya, Ferroelectrics 162, 63 (1994)

[69] M. I. Bichurin, I.A. Kornev, V.M. Petrov, I. Lisnevskaya, Ferroelectrics 204, 289 (1997)

[70] J. Ryu, S. Priya, A.V. Carazo, K. Uchino, H.E. Kim, J. Am. Chem. Soc. 84, 2905 (2001)

[71] S. X. Dong, J.F. Li, D. Viehland, Appl. Phys. Lett. 83, 2265 (2003)

[72] C.W. Nan, M. Li, J.H. Huang, Phys. Rev. B 63, 144415 (2001)

[73] K. Mori, M. Wuttig, Appl. Phys. Lett. 81, 100 (2002)

[74] Shuxiang Dong, Jie-Fang Li, D. Viehland, Appl. Phys. Lett. 83 (2003) 2265

[75] Shuxiang Dong, Jinrong Cheng, J.F. Li, D. Viehland, Appl. Phys. Lett. 83 (2003) 4812

[76] Shuxiang Dong, Jie-Fang Li, D. Viehland, J. Appl. Phys. 96 (2004) 3382

[77] S. X. Dong, J. Y. Zhai, F. Bai, Jie-Fang Li, D. Viehland, Appl. Phys. Lett. 87, 062502 (2005)

[78] J. Y. Zhai, Z. Xing, S. X. Dong, Jie-Fang Li, D. Viehland, Appl. Phys. Lett. 88, 062510 (2006)

[79] C. Kirchhof, M. Krantz, I. Teliban, R. Jahns, S. Marauska, B. Wagner, R. Knochel, M. Gerken, D. Mayners, E. Quandt, Appl. Phys. Lett. Vol. 102, 23, 232905 (2013)

[80] R. Jahns, H. Greve, E. Woltermann, E. Quandt, R. Knochel, Sensors and Actuators A 183, 16-21 (2012)

[81] R. Jahns, H. Greve, E. Woltermann, E. Quandt, R. Knochel, IEEE Trans. Instr. Meas. Vol. 60, No. 8, $2995-$ 3001 (2011)

[82] U. Laletsin, N. Padubnaya, G. Srinivasan, C.P. Devreugd, Appl. Phys. A 78 (2004) 33

[83] Shuxiang Dong, Jie-Fang Li, D. Viehland, J. Cheng, L.E. Cross, Appl. Phys. Lett. 85 (2004) 3534

[84] Y. K. Fetisov, A. A. Bush, K. E. Kamentsev, A. Y. Ostashchenko, G. Srinivasan, IEEE Sensors Journal, 6 (2006) 935

[85] E. Lage, F. Woltering, E. Quandt, D. Mayners, Appl. Phys. Lett. 113, 17C725 (2013)

[86] S. X. Dong, J. Zhai, Jie-Fang Li, D. Viehland, Appl. Phys. Lett. 88, 082907 (2006)

[87] C. Kittel, Phys. Rev. 73, 155 (1948)

[88] A.A. Semenov, S.F. Karmanenko, V.E. Demidov, B.A. Kalinikos, G. Srinivasan, A.N. Slavin, J.V. Mantese, Appl. Phys. Lett. 88, 033503 (2006)

[89] Y. K. Fetisov and G. Srinivasan, Appl. Phys. Lett. 88, 143503 (2006)

[90] V.E. Demidov, B.A. Kalinikos, S.F. Karmanenko, A.A. Semenov, P. Edenhofer, IEEE Trans. Microwave Theory Tech. 51, 2090 (2003)

[91] A. B. Ustinov, G. Srinivasan, B. A. Kalinikos, Appl. Phys. Letter. 90, 031913 (2007)

[92] Y.K. Fetisov, G. Srinivasan, Electronics Letters, vol. 41, No 19, 1066-1067 (2005)

[93] Y.K. Fetisov, G. Srinivasan, Appl. Phys. Lett., 87103502 (2005)

[94] S. Tumanski, Thin Film Magnetoresistive Sensors, IOP publishing (2001) ISBN 0750307021

[95] Sarah M Thompson, J. Phys. D: Appl. Phys. 41 (2008) 093001

[96] E. M. Williams, Design and Analysis of Magnetoresistive Recording Heads, (2001) ISBN 0-471-36358-8

[97] M. Julliere Phys. Lett. 54A (1975) 225

[98] J. C. Slonczewski, Phys. Rev. B 39 (1989) 6995

[99] M. Vopsaroiu, J. Blackburn, M. G. Cain, J. Phys D: Applied Physics, Vol. 40, pp. 5027 (2007)

[100] M. Vopsaroiu, J. Blackburn, A. Piniella, M. G. Cain, Journal of Applied Physics 103, 07 F506 (2008)

[101] Y. Zhang, L. Zheng, C. Deng, J. Ma, Y. Lin, Nan Ce-Wen, Applied Phys. Letters 92, 152510 (2008)

[102] R L Stamps, J. Phys. D: Appl. Phys. 33 (2000) R247-R268

[103] K. O’Grady, L.E.Fernandez-Outon, G.Vallejo-Fernandez, J. Magn. Mag. Mater. 322 (2010) 883-899

[104] Y. Zhou, S.C. Yang, D.J. Apo, D. Maurya, S. Priya, Appl. Phys. Lett. 101, 232905 (2012)

[105] E. Lage, C. Kirchhof, V. Hrkac, L. Kienle, R. Jahns, R. Knöchel, E. Quandt, D. Meyners, Nature Materials, Vol. 11, (6) 523-529, (2012) 
[106] Dudley A. Buck, "Ferroelectrics for Digital Information Storage and Switching." Report R-212, MIT, June 1952

[107] Nian X. Sun, Ming Liu, US Patent WO2013090937 A1 (2013)

[108] Ming Liu and Nian X. Sun, Phil. Trans. R. Soc. A (2014)

[109] A. Kumar, S.R. Katiyar, J.F. Scott, IEEE Transactions on Ferroelectrics and Frequency Control, Vol. 57, Issue 10 (2010)

[110] M. Bibes, A. Barthélémy, Nature Materials 7, 425 - 426 (2008)

[111] M. Gajek, M. Bibes, S. Fusil, K. Bouzehouane, J. Fontcuberta,A. Barthélémy, A. Fert, Nature Materials, vol 6, $296-302$, (2007)

[112] A. Gruverman, D. Wu, H. Lu, Y. Wang, H. W. Jang, C.M. Folkman, M. Ye. Zhuravlev, D. Felker, M. Rzchowski, C.-B. Eom and E. Y. Tsymbal, Nano Lett. 9, 3539 (2009)

[113] E.Y. Tsymbal, A. Gruverman, V. Garcia, M. Bibes, A. Barthélémy, MRS Buletin, Vol. 37,138 - 143 (2012)

[114] R. L. Cummerow, Phys. Rev. vol. 95, No. 1 (1954)

[115] N. López, L.A. Reichertz, K.M. Yu, K. Campman, and W. Walukiewicz, Phys. Rev. Lett. 106, 028701 (2011)

[116] V.M. Fridkin, Ferroelectrics, Volume 53, Issue 1, (1984)

[117] A. M. Glass, D. von der Linde, T. J. Negran, Applied Physics Letters 25, 233, (1974)

[118] W.T.H. Koch, R. Munser, W. Ruppel, P. Würfel, Solid State Communications 17 (7), 847-850 (1975)

[119] V.M. Fridkin, Crystallography Reports 46, 654-658 (2001)

[120] R.V. Schmidt, I.P. Kaminow, Appl. Phys. Lett. Vol 25, No. 8, 458 - 460, (1974)

[121] V.S. Dharmadhikari, W.W. Grannemann, J. Appl. Phys. Vol. 53, 12, 8988 (1982)

[122] K. Yao, B.K. Gan, M.M. Chen, S. Shannigrahi, Appl. Phys. Lett. 87, 212906 (2005)

[123] M. Qin, K. Yao, Y.C. Liang, Appl. Phys. Lett. 93, 122904 (2008)

[124] S. R. Basu, L. W. Martin, Y. H. Chu, M. Gajek, R. Ramesh, R. C. Rai, X. Xu, J. L. Musfeldt, Appl. Phys. Lett. 92, 091905 (2008)

[125] A. J. Hauser, J. Zhang, L. Mier, R. A. Ricciardo, P. M. Woodward, T. L. Gustafson, L. J. Brillson, F. Y. Yang, Appl. Phys. Lett. 92, 222901 (2008)

[126] T. Choi, S. Lee, Y.J. Choi, V. Kiryukhin, S.W. Cheong, Science 324, 63-66 (2009)

[127] R. Nechache, C. Harnagea, S. Licoccia, E. Traversa, A. Ruediger, A. Pignolet, and F. Rosei. Applied Physics Letters, 98, 202902 (2011)

[128] Y. Zhang, X. L. Zhong, M. Vopson, J. B. Wang, Y. C. Zhou, J. Appl. Phys. 112, 014112 (2012)

[129] B. Kundys, M. Viret, D. Colson, D. O. Kundys, Nature Materials 9, 803-805 (2010)

[130] P. Krulevitch, A. P. Lee, P. B. Ramsey, J. C. Trevino, J. Hamilton, M. A. Northrup, J. Microelectromech. Systems 5 (1996) 270

[131] V. Srivastava, Y. Song, K. Bhatti, R. D. James, Adv. Energy Mater. 1, 97-104 (2011)

[132] V. Srivastava, X. Chen, R. D. James. Appl. Phys. Lett. 97, 014101 (2010)

[133] Bernard D. H. Tellegen , Philips Res. Rept. 3, 81-101 (1948)

[134] J. Zhai, J. Gao, C. De Vreugd, J. Li, D. Viehland, A. V. Filippov, M. I. Bichurin, D. V. Drozdov, G. A. Semenov, S. X. Dong, Eur. Phys. J. B 71, 383-385 (2009)

[135] W.F. Giauque, D.P. MacDougall, Phys. Rev. 43 (9), 768 (1933)

[136] K.A. Gschneidner, V.K. Pecharsky. International Journal of Refrigeration, 31, 945-961, (2008)

[137] H. Ishimoto, N. Nishida, T. Furubayashi, M. Shinohara, Y. Takano, Y. Miura, and K. Ono, J. Low Temp. Phys. 55, 17 (1984)

[138] K. A. Gschneidner, V. K. Pecharsky, A. O. Tsokol, Rep. Prog. Phys. 68, 1479-1539 (2005)

[139] J.F. Scott, Annu. Rev. Mater. Res. 41, 1-12 (2011)

[140] E. Bonnot, R. Romero, L. Man osa, E. Vives, A. Planes, Phys. Rev. Lett. 100 (2008) 125901

[141] T. Castan, A. Planes, A. Saxena, Phys. Rev. B, 85, 144429 (2012)

[142] X. Moya, L. E. Hueso, F. Maccherozzi, A. I. Tovstolytkin, D. I. Podyalovskii, C. Ducati, L. C. Phillips, M. Ghidini, O. Hovorka, A. Berger, M. E. Vickers, E. Defay, S. S. Dhesi \& N. D. Mathur, Nature Materials, 12, 52-58 (2013)

[143] M. Vopson, Solid State Communications 152, 2067-2070 (2012)

[144] S. Fahler, U. K. Roßler, O. Kastner, J. Eckert,G. Eggeler, H. Emmerich, P. Entel, S. Muller,E. Quandt, K. Albe, Adv. Eng. Mat., 14, No. 1-2 (2012)

[145] H. Meng, B. Li, W. Ren, Z. Zhang, Physics Letters A, 377, 567-571, (2013)

[146] S. Lisenkov, B. K. Mani, C-M. Chang, J. Almand, I. Ponomareva, Phys. Rev. B 87, 224101 (2013)

[147] M.M. Vopson, J. Phys. D: Appl. Phys. 46 (2013) 345304

[148] A. Midya, S.N. Das, P. Mandal, Phys. Rev. B 84 (2011) 235127

[149] J. Ma, Z. Li., Y. Lin, C.W. Nan, J. Magn. Magn. Mater. 323, 101-103 (2011)

[150] L. Y. Fetisov, Y. K. Fetisov, G. Sreenivasulu, G. Srinivasan, J. Appl. Phys. 113, 116101 (2013) 
[151] Shuxiang Dong, J. F. Li, D. Viehland, J. Cheng, L. E. Cross, Appl. Phys. Lett. Vol. 85, No. 16, 35343536 (2004)

[152] V. Bedekar, R. A. Islam, H. Kim, M. I. Bichurin, S. N. Ivanov, Y. J. Pukinski, S. Priya, Eur. Phys. J. B 71, 387-392 (2009)

[153] S. X. Dong, J.F. Li, D. Viehland, Appl. Phys. Lett. 85, 2307 (2004)

[154] N.G. Stephen, Journal of Sound and Vibration, 293, 409-425 (2006)

[155] H.U. Kim, W.H. Lee, H.V. Rasika Dias, S. Priya, IEEE TUFFC, vol. 56, No. 8 (2009)

[156] E. Lefeuvre, A. Badel, C. Richard, L. Petit, D. Guyomar, Sensors and Actuators, A, 126, 405-416 (2006)

[157] M Deterre, E Lefeuvre and E Dufour-Gergam, Smart Mater. Struct. 21 (2012) 085004

[158] C. A. Kitio Kwuimy, G. Litak, M. Borowiec, C. Nataraj, Appl. Phys. Lett. 100, 024103 (2012)

[159] L. Wang, F. G. Yuan, Smart Mater. Struct. 17 (2008) 045009

[160] M. Zucca, O. Bottauscio, C. Beatrice, F. Fiorillo, IEEE Trans. Magn., Vol. 47, No. 10, (2011)

[161] T. Lafont, L. Gimeno, J. Delamare, G. A. Lebedev, D. I. Zakharov, B. Viala, O. Cugat, N. Galopin, L. Garbuio, O. Geoffroy, J. Micromech. Microeng. 22 (2012) 094009

[162] H.K.D. Kim, L.T. Schelhas, S. Keller, J.L.Hockel, S.H. Tolbert, G.P. Carman, Nano Letters, 13, 884-888 (2013)

[163] M. Vopson, E. Zemaityte, M. Spreitzer, E. Namvar, J. Appl. Phys. Vol. 116, 113910 (2014)

[164] G. Caruntu, A. Yourdkhani, M. Vopsaroiu, G. Srinivasan, Nanoscale, 4 (10). pp. 3218-3227 (2012)

[165] A. Kulkarni, K. Meurisch, I. Teliban, R. Jahns, T. Strunskus, A. Piorra, R. Knochel, F. Faupel, Appl. Phys. Lett., vol. 104, 022904 (2014)

[166] M.O. Le, F. Belhora, A. Cornogolub, P.J. Cottinet, L. Lebrun, A. Hajjaji, J. Appl. Phys., vol. 115, 194103 (2014) 